\title{
Vortex induced vibrations at high Reynolds numbers on circular cylinders M. Belloli ${ }^{\text {a }}$, S. Giappino ${ }^{\text {a,* }}$, S. Morganti ${ }^{\text {b }}$, S. Muggiasca ${ }^{\text {a }}$, A. Zasso ${ }^{\text {a }}$ \\ a Politecnico di Milano, Dipartimento di Meccanica, Via La Masa 1, 20126 Milano, Italy \\ ${ }^{\mathrm{b}}$ Università degli Studi di Pavia, Dipartimento di Ingegneria Civile e Architettura (DICAr), Via Ferrata 1, 27100 Pavia, Italy
}

\section{ABSTRACT}

The Reynolds Number is a fundamental parameter in defining vortex shedding from circular cylinders. Most of the studies of vortex induced vibrations are at subcritical and critical Reynolds Number values and there is a need to understand how the Reynolds Number affects these oscillations. An experimental set-up was created in a large wind tunnel to study vortex induced vibrations at high Reynolds Numbers. The circular cylinder model had a diameter of $0.72 \mathrm{~m}$, allowing Reynolds Numbers up to $6 \cdot 10^{5}$ to be reached. The cylinder had a mass ratio value of 35 , quite low with respect to other tests in air because of its large dimensions but still high compared to the mass ratios obtainable testing in water. By increasing the model's surface roughness it was possible to reach the postcritical flow regime that produces coherent and highly organized vortex shedding. The set-up permitted the simultaneous measurement both of the instantaneous fluid force calculated integrating surface pressure distribution and the model oscillation. The article describes the results of the tests and compares the characteristics of vortex induced vibrations in postcritical flow regime with subcritical ones.

\section{Introduction}

Vortex induced vibrations of circular section structures are a wellknown and widely studied phenomenon and can occur in many engineering applications. The topic has been widely reviewed (Sarpkaya, 1979; Bearman, 1984; Sarpkaya, 2004; Williamson and Govardhan, 2004), and there are several contributions in the literature on the subject. Experimental analyses have been carried out by different researchers using rigid models suspended on springs, flexible models or also externally excited cylinders (Williamson and Roshko, 1988; Brika and Laneville, 1993; Khalak and Williamson, 1999; Carberry et al., 2001). The aim of all these studies was to define fluid-structure interaction in terms of lock-in range, vibration amplitudes, force applied by the fluid to the cylinder and power provided by the fluid flow to the mechanical system, (Diana and Falco, 1971; Brika and Laneville, 1996).

The control parameters of the fluid-elastic interaction depend on the structure considered (dimensions, mass, damping ratio, natural frequency and surface roughness) and the fluid (velocity, density, viscosity, turbulence intensity). In spite of this large set of physical quantities, a reduced set of non-dimensional parameters was defined

* Corresponding author.

E-mail address: stefano.giappino@polimi.it (S. Giappino). to describe the characteristics of free motion response: the Scruton Number or mass damping ratio, mass ratio and velocity-ratio. As is well known from studies using still cylinders, (Schewe, 1983; Zdravkovich, 1997), the Reynolds Number too strongly influences interaction between the cylinder and the incoming flow. In particular, the vortex shedding phenomenon is well defined in the subcritical Reynolds Number range, where it is markedly periodic and the wake has a narrow band spectrum. In the critical region, the phenomenon has reduced magnitude, the harmonic content of the wake covers a wide frequency range and sometimes disappears. Regular vortex shedding can be seriously disrupted if the separation line is not straight (Bearman, 1984), as in the critical region. Nevertheless, recent studies observed vortex induced vibrations in the critical region (Ding et al., 2004; Raghavan and Bernitsas, 2011; Pastò, 2008), but further analysis are necessary to completely define the phenom-enon in this condition. (Roshko, 1961) found that vortex shedding reappears at higher Reynolds Numbers, in what is called the postcritical flow regime. In this condition, laminar-turbulent transi-tion occurs within the attached boundary layer and a straight separation line is reestablished. Surface roughness affects vortex shedding owing to its effects on the body's surface characteristics and consequently on the boundary layer (Buresti, 1981). Many studies (Buresti, 1981; Zdravkovich, 1997) reported that the presence of roughness on the cylinder's surface anticipates the laminar-turbulent transition in the boundary layer and also the 'drag crisis region' at 
smaller Reynolds Numbers. Hence surface roughness can be used to simulate higher Reynolds Numbers, as suggested by (ESDU, 1986). ESDU introduces the effective Reynolds Number parameter that is a modified Reynolds Number function of incoming turbulence intensity and surface roughness. Defining the effective Reynolds Number is difficult mainly because of the difficulties of quantifying surface roughness, but it is generally assumed that an increase in roughness corresponds to an increase in the Reynolds Number.

It is expected that the roughness parameter also greatly influences vortex induced vibrations.

The influence of the Reynolds Number on vortex shedding has been extensively studied on fixed cylinders, while only a few studies have investigated its effects on cylinders free to move in a cross-wind direction. A study by Govardhan and Williamson (2006) highlights the dependence of the maximum oscillation amplitude on the Reynolds Number. In particular, Eq. (1) presented in (Govardhan and Williamson, 2006) defines the maximum non-dimensional amplitude $\left(A^{*}\right)$ as a function of the Reynolds Number and mass damping ratio $(\alpha$, see Table 1$)$ :

$A^{*}=\left(1-1.2 \alpha+0.3 \alpha^{2}\right) \log \left(0.41 \mathrm{Re}^{0.36}\right)$

This relation has been verified up to Reynolds Numbers relative to the subcritical region: for higher Reynolds Numbers (critical region) only a few data are available. Ding et al., (2004); ASME (2008) and Raghavan and Bernitsas (2011) performed tests at Reynolds Numbers respectively up to $2.510^{5}$ and $1.510^{5}$. In particular, Raghavan and Bernitsas observed unexpectedly high oscillation amplitudes comparing their results with Eq. (1). No contribution on VIV at postcritical Reynolds Number has been found.
This article investigates the behaviour of a circular cylinder under flow conditions at high Reynolds Numbers, that is, in the critical and postcritical flow field conditions. Postcritical Reynolds Numbers were obtained by using a large model, of diameter 0.72 $\mathrm{m}$, and increasing the surface roughness.

The results are compared with the data previously obtained in the subcritical flow regime using a similar set-up described in (Zasso et al., 2008; Belloli et al., 2012).

\section{Model and experimental set-up}

The research was carried out in the Politecnico di Milano boundary layer wind tunnel. The test section was $4 \mathrm{~m}$ high $\left(H_{\mathrm{ts}}\right)$ and $14 \mathrm{~m}$ wide $\left(L_{\mathrm{ts}}\right)$ and its turbulence intensity was $I_{\mathrm{u}}=2 \%$. The integral length scale in the flow direction $\mathrm{L}_{\mathrm{u}}^{\mathrm{x}}$ was $0.2 \mathrm{~m}$. As observed in a previous study in the Politecnico di Milano wind tunnel (Zasso et al., 2005), the turbulence level of the boundary layer test section anticipates critical drag at about $R e=2 \cdot 10^{5}$, but does not affect the shape of the drag curve and the Strouhal Number trend. Variations in the horizontal wind velocity profile are lower than $3 \%$.

The experimental set-up was designed to perform cross-wind free motion tests and to study the model's dynamics and the related fluid dynamic features. The same set-up allowed the model to be constrained for fixed cylinder testing as described in (Belloli et al., 2007).

The model was a smooth carbon fibre cylinder $3.3 \mathrm{~m}$ long and 0.72 $\mathrm{m}$ in diameter. Two large end-plates $\left(D_{\text {end-plates }} / D=2\right)$ were placed at the ends of the cylinder to create bidimensional flow conditions. The diameter of the end plates was a compromise between

Table 1

Nomenclature.

\begin{tabular}{|c|c|c|c|}
\hline$\zeta$ & Nondimensional structural damping (damping to critical damping ratio) & $\bar{p}_{\mathrm{s}}$ & Reference static pressure \\
\hline$D$ & Cylinder diameter & $\rho$ & Air density \\
\hline$f$ & Actual frequency of oscillating cylinder in the flow & $\operatorname{Re}$ & Reynolds Number, $\operatorname{Re}=\frac{\rho U D}{\mu}$ \\
\hline$f_{s}$ & Structural frequency in still air & Sc & Scruton Number, $S c=\frac{2 \pi m_{L} \zeta}{\rho D^{2}}$ \\
\hline$f^{*}$ & Frequency ratio, $f^{*}=\frac{f}{f_{s}}$ & St & Strouhal Number \\
\hline$L$ & Cylinder length & $U$ & Incoming flow velocity \\
\hline $\mathrm{L} / \mathrm{D}$ & Aspect ratio & $U_{\mathrm{St}}$ & Strouhal velocity, $U_{S t}=\frac{f_{S} D}{S t}$ \\
\hline$m_{L}$ & Mass per unit length & $f_{\mathrm{St}}$ & Strouhal frequency, $f_{S t}=\frac{S t U}{D}$ \\
\hline$m^{*}$ & Mass ratio, $m^{*}=\frac{m_{E}}{\pi \rho D^{2} / 4}$ & $U^{*}$ & Velocity ratio, $U^{*}=\frac{U}{U_{S t}}$ \\
\hline$\alpha$ & $m^{*} \zeta$ mass damping ratio & $z$ & Cross-flow cylinder amplitude \\
\hline$M$ & Air viscosity & $z / D$ & Dimensionless amplitude \\
\hline$P$ & Actual pressure measured at each tap & & \\
\hline
\end{tabular}

a

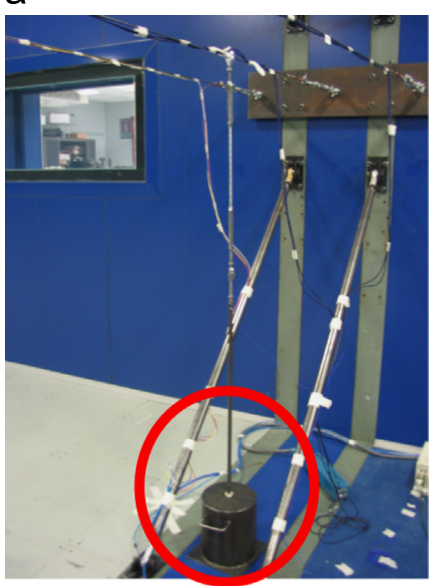

b

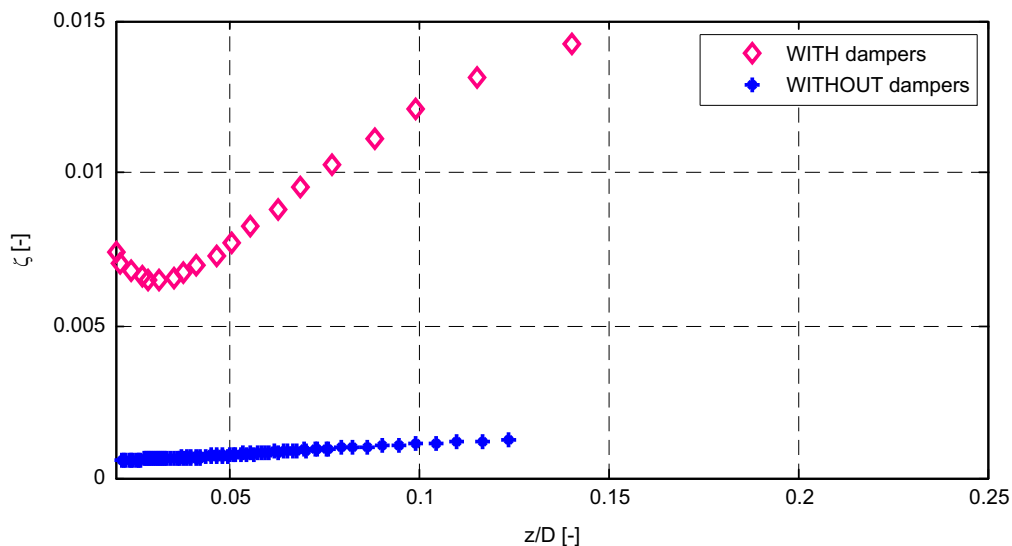

Fig. 1. (a) Oil damper (b) Structural non-dimensional damping as a function of non-dimensional oscillation amplitude. 
two different requirements: having end plates large enough to ensure bidimensional flow but small enough to avoid interference and undesired vibrations in the vertical motion of the cylinder. Moreover, in a previous study (Zasso et al., 2005), it was observed that the influences of the extremities is more important in the subcritical region, but less important in the critical and postcritical regions.

Aspect ratio $(L / D=3)$ effects were also considered: Buresti, Martini 1980 notice that for small aspect ratios such as 3, a small increase in the suction peaks is observed, while the drag coefficient and the Strouhal Number showed no significant differences.

The cylinder was linked to the wind tunnel walls using three tensioned steel wires (see Fig. 4). The constraining system acts like a spring in the vertical direction, with constant stiffness for the oscillation amplitudes reached during the tests. Any coupling of inline and torsional motion was avoided by a sufficiently wide separation of the horizontal, torsional and vertical frequencies.

The structural frequency in still air $f_{\mathrm{s}}$ of the suspended model was $f_{\mathrm{s}}=1.95 \mathrm{~Hz}$. The structural damping ratio, $\zeta$, was obtained from decay tests in still air and depends on the oscillation amplitude, as shown in Fig. 1 and as reported in (Brika and Laneville, 1996) and (Diana et al.,

Table 2

Model characteristics.

\begin{tabular}{lll}
\hline Mechanical characteristics & $\begin{array}{l}\text { Subcritical } \\
\text { Reynolds }\end{array}$ & $\begin{array}{l}\text { Postcritical } \\
\text { Reynolds }\end{array}$ \\
\hline Diameter $D[\mathrm{~m}]$ & 0.2 & 0.72 \\
Length $[\mathrm{m}]$ & 2 & 3.3 \\
Mass per unit length $[\mathrm{kg} / \mathrm{m}]$ & 6.5 & 17 \\
Aspect ratio $L / D$ & 10 & 4.6 \\
Mass ratio $m^{*}$ & 170 & 35 \\
Frequency in still air $[\mathrm{Hz}]$ & 3.1 & 1.95 \\
Structural damping ratio $\zeta$ at $z / D=0.1:$ & & \\
Without dampers & $\zeta_{1}=0.6 \cdot 10^{-3}$ & $1.1 \cdot 10^{-3}$ \\
With dampers & $\zeta_{2}=2.5 \cdot 10^{-3}$ & $1.2 \cdot 10^{-2}$ \\
& $\zeta_{3}=4.1 \cdot 10^{-3}$ & \\
Scruton Number at $z / D=0.1:$ & & 0.19 \\
Without dampers & $\mathrm{Sc}_{1}=0.5$ & 2.11 \\
With dampers & $\mathrm{Sc}_{2}=2.18$ & \\
& $\mathrm{Sc}_{3}=3.6$ & \\
$m^{*} \zeta$ at $z / D=0.1:$ & & 0.038 \\
Without dampers & 0.1 & 0.42 \\
With dampers & 0.43 & \\
Surface & 0.7 & \\
Aerodynamic characteristics & $\mathrm{Smooth}^{-3}$ & \\
Strouhal number & & 0.17 \\
Strouhal velocity $[\mathrm{m} / \mathrm{s}]$ & 0.18 & \\
Nominal Reynolds number $($ at Strouhal & $4.6 \cdot 10^{4}$ & \\
$\quad$ velocity) & & \\
\hline
\end{tabular}

a

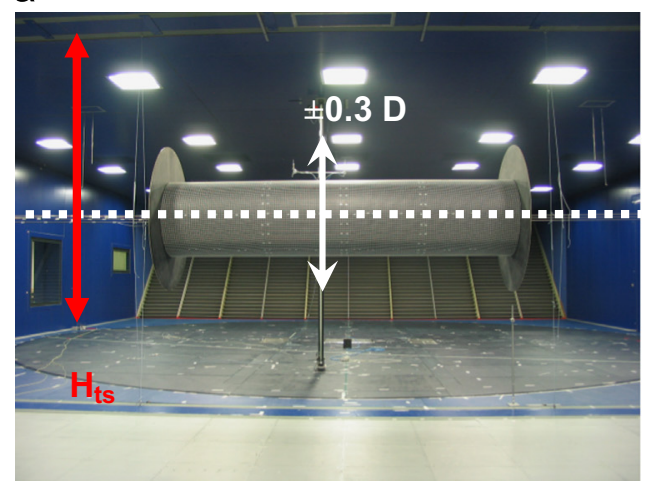

2006). Damping was increased to produce different non-dimensional damping values and, consequently, different Scruton Numbers. It is well known that the mass damping ratio and the Scruton Number are the same parameter despite of a constant factor. The set-up designed, has very low values of non-dimensional damping without dampers. To limit oscillation amplitudes for safety reasons, tests were performed at high damping values to limit the maximum amplitude at $0.22 \mathrm{D}$ (where $\mathrm{D}$ is the cylinder diameter). Oil dampers were used to control the damping factor. They consisted of perforated plates moving in oil-filled tanks: oil flowing through the holes in the plates created forces proportional to the plates' velocity. Damping forces were transmitted to the cylinder connecting the dampers to the oscillating system, as shown in (Fig. 1 (a)). The dampers were effective but had nonlinear behaviour; damping was a function of oscillation amplitude, as shown in Fig. 1 (b) where non-dimensional damping is shown as a function of non-dimensional cylinder oscillation. The two curves were obtained from decay tests in still air with and without dampers.

The Scruton Numbers reported in Table 2 have nominal values calculated using the damping ratio at $z / D=0.1$.

The main model data are summarised in Table 2 (postcritical Reynolds set-up column).

The blockage of the model in the test section, that is, the ratio between the frontal area of the cylinder and that of the test section, is $4.4 \%$ : no correction was made on the aerodynamic coefficients. In (Zdravkovich, 1997) blockage lower than $10 \%$ is considered small, so it may be ignored.

Wall proximity effects were carefully investigated (Belloli et al., 2007) and were evaluated as negligible. These effects were studied in preliminary investigations with the cylinder in the fixed condition, installing it in both the vertical and horizontal positions (see Fig. 2). In the vertical position, the ratio between the cylinder's diameter $D$ and the test section's width $\left(L_{\mathrm{ts}}\right)$ was $5 \%\left(D / L_{\mathrm{ts}}\right)$. In the horizontal position, the ratio between the cylinder diameter and the tunnel height $\left(H_{\mathrm{ts}}\right)$ was $18 \%\left(D / H_{\mathrm{ts}}\right)$. Aerodynamic forces and pressure distributions obtained in these two conditions were very similar, showing that proximity effects can be considered irrelevant as seen in Fig. 3(a), where mean drag and lift coefficient as a function of Reynolds Number are shown. The curves obtained in the different test conditions are very similar.

The model was tested in the fixed condition and horizontal arrangement $0.3 \mathrm{D}$ from the centre of the test section (Fig. 2(a)). This condition reproduced the position reached by the cylinder during VIV tests and permitted verification of the wall proximity effect on the aerodynamic forces and pressure distributions. The tests confirmed that the conditions chosen for the dynamic tests were not affected by boundary effects, as shown in Fig. 3(b).

The model was fitted with 126 pressure taps distributed in three rings (Fig. 5).

b

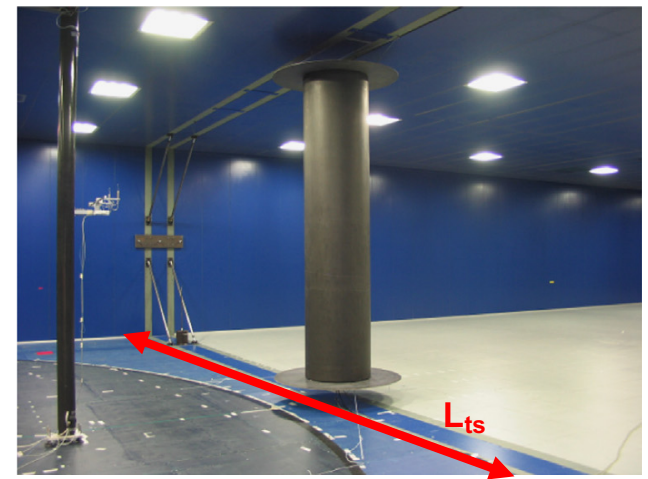

Fig. 2. Fixed cylinder test configurations: (a) horizontal arrangement in the middle of the test section and 0.3D from the middle, (b) vertical arrangement. 

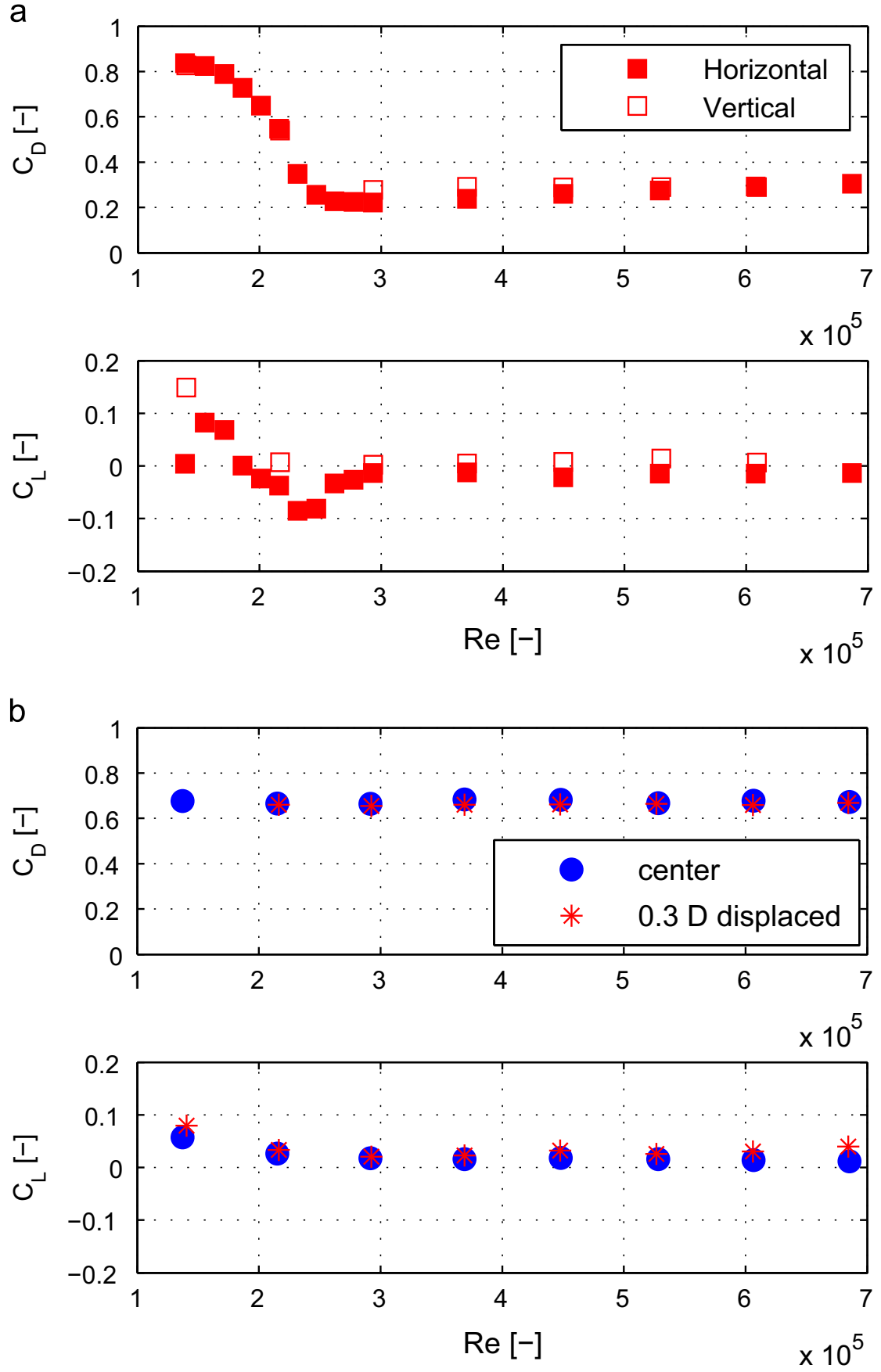

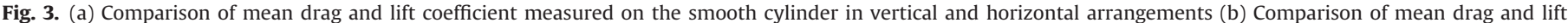
coefficient measured on the rough cylinder in the middle of the test section and 0.3D from the middle.

The taps were connected to high sample rate pressure scanners, permitting high resolution measurements of the pressure field in the time domain.

The pressure value is expressed by the non-dimensional coefficient $C_{P}$ normalized on the mean wind dynamic pressure measured by a pitot tube (all the quantities are reported in Table 1)

$C_{\mathrm{P}}(t)=\frac{p(t)-\bar{p}_{\mathrm{s}}}{(1 / 2) \rho U^{2}}$

The drag and lift forces were evaluated by integrating the surface pressure on each ring:

$C_{\mathrm{D}}(t)=\frac{1}{2} \int_{0}^{2 \pi} C_{\mathrm{P}}(t) \cdot \cos (\vartheta) d \vartheta=\frac{1}{2} \sum_{i} C_{\mathrm{pi}}(t) \cdot \cos \left(\vartheta_{i}\right) \Delta \vartheta_{i}$

$$
C_{\mathrm{L}}(t)=\frac{1}{2} \int_{0}^{2 \pi} C_{\mathrm{P}}(t) \cdot \sin (\vartheta) d \vartheta=\frac{1}{2} \sum_{i} C_{\mathrm{pi}}(t) \cdot \sin \left(\vartheta_{i}\right) \Delta \vartheta_{i}
$$

where $\vartheta$ is the angle between the pressure tap and wind velocity direction (Fig. 5). Note that integrating only the surface pressures excludes friction forces, but these can be neglected at these Reynolds Number values (Zdravkovich, 1997).

Following these conventions, the drag coefficient represents the force component in the direction of the undisturbed flow velocity, while the lift coefficient is the force component perpendicular to the flow (see Fig. 5).Vertical cylinder motion was monitored by two accelerometers placed at the model end sections. A third horizontal accelerometer was added to check possible in-line vibrations: during the tests the model showed purely vertical motion. Two 
high frequency response three-components anemometers (4hole pressure probes) were placed downstream of the cylinder to measure vortex shedding frequency.

The results obtained were compared with data from a previous research conducted in the Politecnico di Milano wind tunnel on a smaller cylinder at subcritical Reynolds Numbers (Zasso et al., 2008; Belloli et al., 2012). The previous set-up was similar to the present one: the model was a smooth acrylic cylinder with a diameter of $0.2 \mathrm{~m}$, elastically suspended from the wind tunnel walls. Table 2 gives the mechanical and aerodynamic characteristics of both set-ups.

\section{Experimental results}

The main results of the experimental tests in terms of both steady state and transient response are reported below. These data were compared to the ones obtained in a previous study performed at lower Reynolds Numbers (Zasso et al., 2008; Belloli et al., 2012). The results of tests on the fixed cylinder are shown to discuss the effective Reynolds Number of the set-up. All the tests were per-formed on the cylinder in the horizontal position after verifying wall proximity effects as described in the previous section. The aero-dynamic forces presented in the article derive from integration of pressure distributions on the central ring. Very similar results were obtained in correspondence with the other two rings.

\subsection{Fixed cylinder at high Reynolds numbers}

Before investigating VIV, a preliminary study was carried out on the fixed model to define the different Reynolds flow regimes encountered by the cylinder (Belloli et al., 2007). Fig. 7 shows the drag coefficient as a function of the Reynolds Number: for the smooth surface condition, drag is about 0.85 for $R e=10^{5}$ dropping to 0.3 at $R e=2.510^{5}$. The drag crisis highlights that the cylinder is in the critical Reynolds regime. Fig. 7 also shows the trend of the

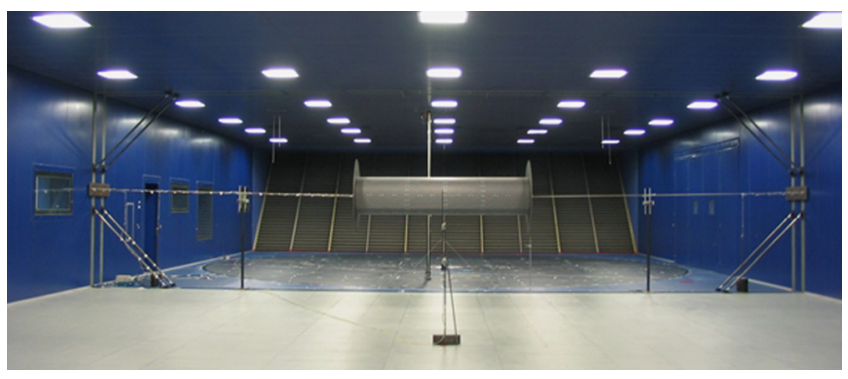

Fig. 4. Cylinder model in the wind tunnel ( $D=0.72 \mathrm{~m}$ - Rough configuration). standard deviation of the lift coefficient $\left(C_{\mathrm{L}}^{\prime}\right)$. Values of $\mathrm{C}_{\mathrm{L}}^{\prime}$ are high in the precritical region and fall with the drag crisis. The values measured after the drag crisis are in agreement with the data scatter reported by (Zdravkovich, 1997).

Moreover, in the precritical regime, at $R e=1.410^{5}$, vortex shedding is still visible in the wake: in Fig. 8(a), the PSD of the vertical component of the wind velocity measured by an anemometer placed in the wake shows a clear peak at the Strouhal frequency (see Table 1). However, at higher Reynolds Numbers regular vortex shedding disappears and the wake shows a broad-band spectrum (Fig. 8(b)).

The mean lift coefficient (see Fig. 7) showed quite small values in the critical region compared with those observed in the literature for similar Reynolds Numbers (Schewe, 1983). This is because pressure distribution does not show significant asymme-tries and the noted 'single bubble' was not observed. As high-lighted in (Zdravkovich, 1997), this regime is very sensitive and may not appear because of a parameter such as flow turbulence.

To increase the effective Reynolds Number (ESDU, 1986), the model's surface roughness was increased by applying a nylon net (15 $\mathrm{mm} \times 15 \mathrm{~mm}$ mesh, $1.5 \mathrm{~mm}$ wire diameter, see Fig. 6) that created a $\mathrm{k} / \mathrm{D}$ ratio of $0.2 \%$ ( $\mathrm{k}$ is the height of the roughness, assumed to be equal to the diameter of the net wire). Surface roughness shifts the drag crisis to lower Reynolds Numbers, allowing high effective Reynolds Number to be attained without increasing wind velocity. Fig. 7 shows that in rough surface conditions the drag coefficient increases with respect to the minimum reached in the critical regime, and is constant throughout the velocity range investigated. It is hard to define the effective Reynolds Number, but it can be assumed that the cylinder is in a postcritical flow regime. Also, the $C_{\mathrm{L}}^{\prime}$ shows a constant trend throughout the Reynolds Number range tested, but its value is comparable with the one reached in the critical region. The results obtained for lift coefficient standard deviation are in agreement with the data collected in (Zdravkovich, 1997) for similar values of $k / D$.

The mean lift coefficient (see Fig. 7) is constant and close to zero, as expected in a regime with symmetrical mean pressure distributions.

Vortex shedding in the rough model reappears in the wake spectrum (Fig. 8(c)): this behaviour can be related to the postcritical
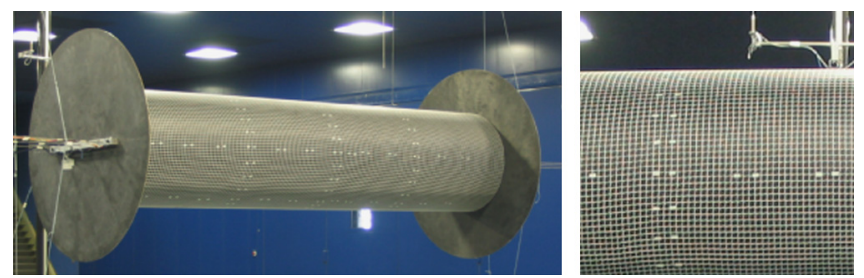

Fig. 6. Cylinder model covered by the net and net detail.
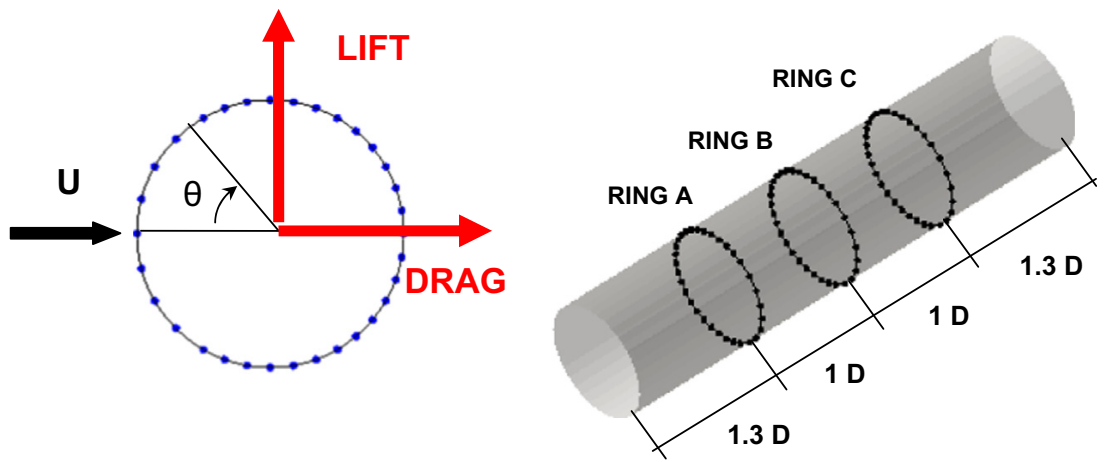

Fig. 5. Pressure tap layout on the model. 
flow regime defined by (Roshko, 1961). The Strouhal Number can be estimated by the wake spectrum in subcritical and postcritical ranges and is equal to 0.18 and 0.17 respectively. The value obtained in postcritical regime is a little lower than the values given in the literature of between 0.19 and 0.24 (Buresti, 1981; Zdravkovich, 1997). At critical Reynolds Numbers the broad band spectrum of the wake makes it difficult to identify the Strouhal Number precisely.
In conclusion, modifying surface roughness allowed investigations of VIV to be extended to postcritical flow conditions.

\subsection{Oscillating cylinder}

Fluid-structure interaction with an oscillating cylinder can usually be described in terms of lock-in range, vibration amplitudes and force exerted by the fluid on the cylinder.
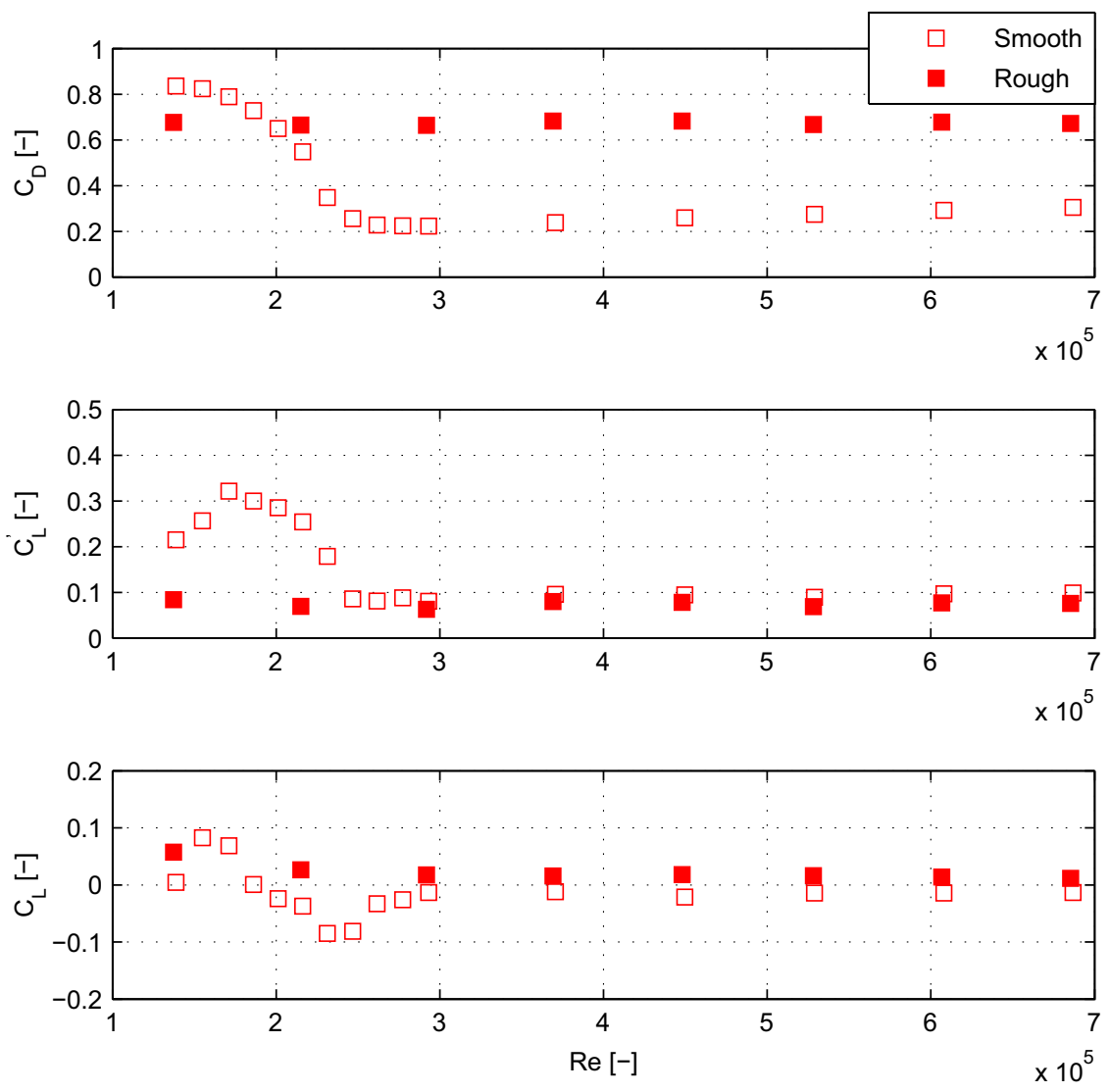

Fig. 7. Mean drag, lift standard deviation and mean lift coefficients as a function of Reynolds Number for smooth and rough surface cylinder.
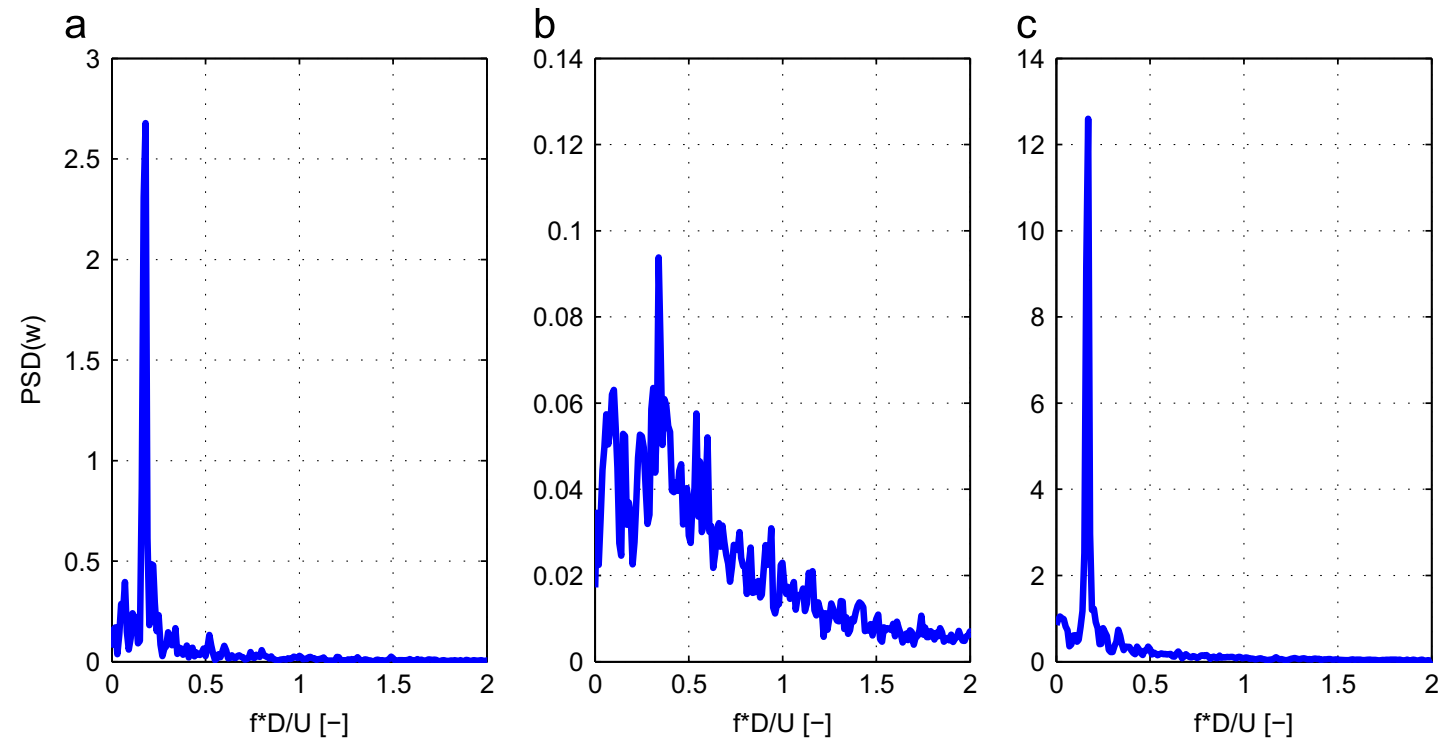

Fig. 8. Wake PSD for different Reynolds Number and surface conditions: (a) $\operatorname{Re}=1.4 \cdot 10^{5}-\operatorname{Smooth}(\mathrm{b}) \operatorname{Re}=6.1 \cdot 10^{5}-\mathrm{Smooth}(\mathrm{c}) \operatorname{Re}=6.1 \cdot 10^{5}-\mathrm{Rough}$. 
Two different kinds of tests were performed to study VIV on cylinders:

- progressive regime tests: steady state conditions were created at a fixed wind speed. The wind velocity was changed by small steps up and down

- build-up tests: the cylinder was released from rest at a fixed wind velocity, in lock-in range, to monitor the vibration transient (increasing amplitude)

\subsubsection{Progressive regimes}

During progressive regime tests, the steady state response was studied for different incoming flow velocities: each regime condition was reached starting from the previous one and increasing or decreasing wind speed in small steps. All the results from the 0.72 $\mathrm{m}$ diameter model were obtained with the rough surface, that is, in the postcritical Reynolds Number range.

Fig. 9 shows the main results of these tests as a function of the velocity ratio $U / U_{S t}$ where $U_{S t}$ is the Strouhal velocity, that is, the wind velocity that synchronizes the vortex shedding frequency with the cylinder's natural frequency (see Table 1). Fig. 9(a) shows the non-dimensional amplitude when increasing (Up) and decreasing (Down) the incoming wind speed. The maximum displacement $z / D=0.22$ was reached for velocity ratios close to 1 and it was limited by introducing additional damping ( $S c=0.42$ at $z \mid D=0.1$ as reported in Table 2). As explained above, no test at low Scruton Numbers was performed to avoid damaging the set-up. The maximum oscillation amplitude reached was lower than expected from Eq. (1) for the declared Scruton Number: this can be partially justified by the non-linear dependence of damping on oscillation amplitude. It is also to observe that Williamson's equation has been obtained analysing experimental data in subcritical Reynolds Number range.

The maximum value was reached by increasing wind velocity, while lower amplitudes were observed at the same $U / U_{\mathrm{St}}$ with decreased wind velocity: this hysteresis phenomenon is well documented in literature (Khalak and Williamson, 1999) and can involve a larger range of velocity ratios. Khalak and Williamson have identified different branches in the steady state response of a cylinder in free motion conditions: Initial Branch, Upper Branch and Lower Branch. On the basis of their studies, hysteresis can appear when the cylinder passes from one branch to another. In the curve shown in Fig. 9(a), it is possible to identify two branches comparable with Initial and Lower branches: the first part of the response curve, $U / U_{\mathrm{St}}<1$ can be related to the Initial Branch, while the second part, $U / U_{\mathrm{St}}>1$ can be related to the Lower Branch. The Upper Branch, with its high oscillation amplitudes, cannot be reached in this mass damping configuration. It must be pointed

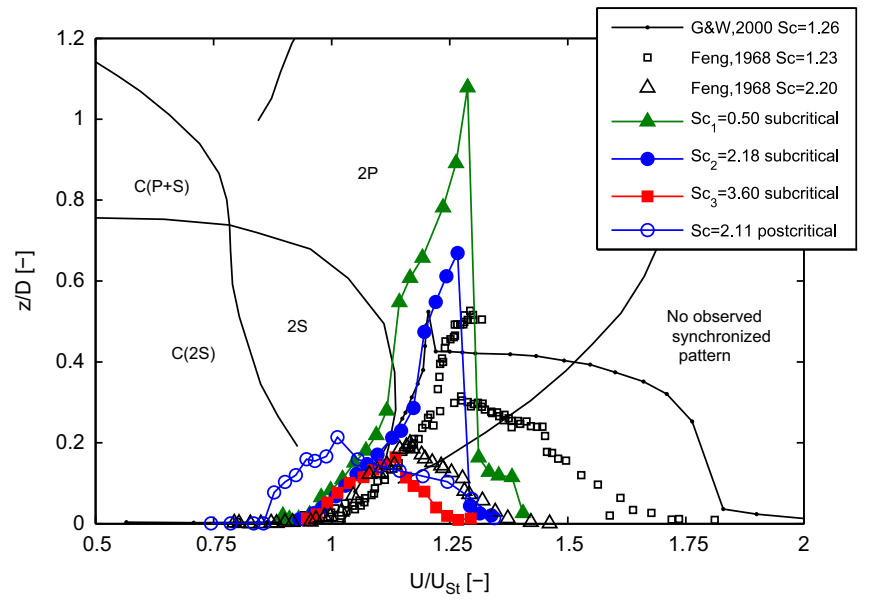

Fig. 10. Free vibration cylinder response in the plane $\mathrm{z} / \mathrm{D}$ vs. $U / U_{\mathrm{St}}$. Zasso progressive regimes (Zasso et al., 2006) data have been compared with Feng results (Feng, 1968) and Govardhan \& Williamson results $\left(m^{*}=320, m^{*} \zeta=0.256, \operatorname{Re}=1250\right)$ (Govardhan and Williamson, 2000).

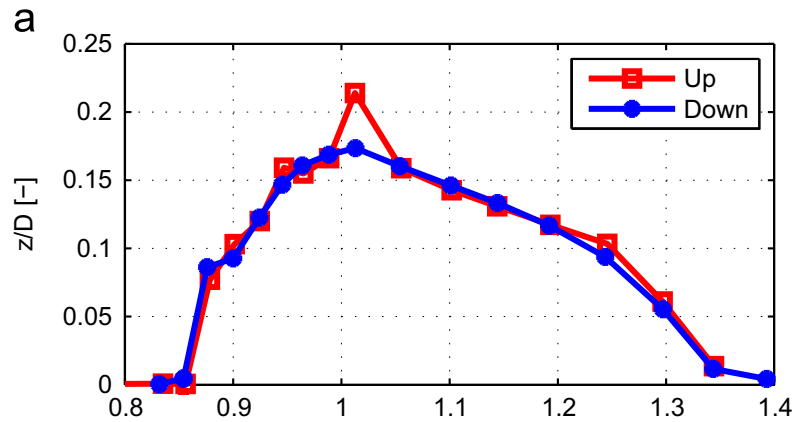

b

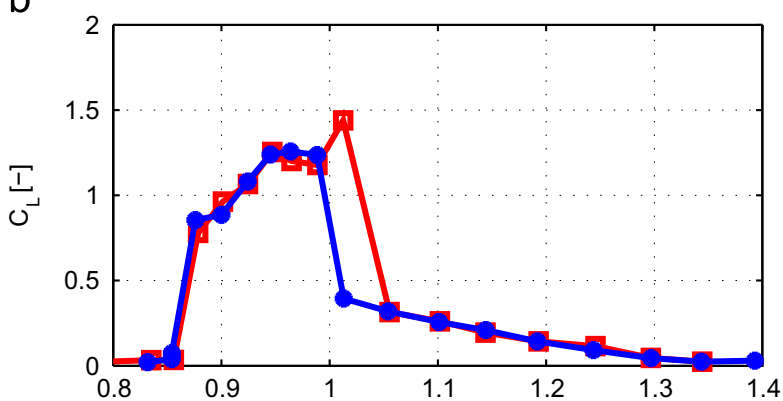

C

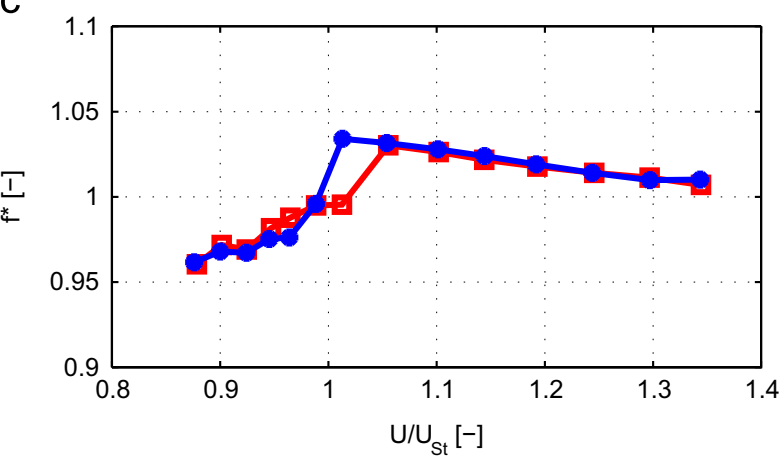

d

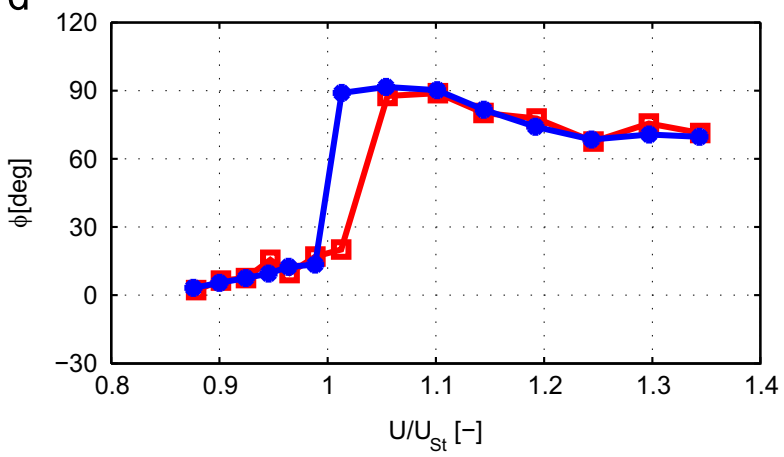

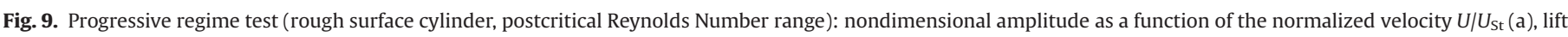

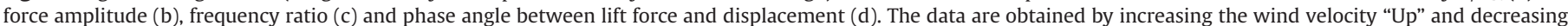
“Down”. 
out that that Upper Branch could perhaps be reached in small damping conditions. The analogies between these two observed branches and the ones identified by (Khalak and Williamson, 1999) are more evident considering the lift force coefficient both in terms of magnitude and phase.

Fig. 9(b and d) describes the characteristics of the fluctuating lift force at the oscillation frequency in terms of magnitude and relative force-displacement phase. The different characteristics of the two branches can be seen: in the first, the lift force reaches quite high values, $C_{\mathrm{L}} \approx 1.5$, and the phase is small. In the second, force diminishes but the phase, close to $90 \mathrm{deg}$, is very effective in introducing power into the system. The behaviour observed is in agreement with the description of the Initial and Lower Branches in (Khalak and Williamson, 1999). In particular, the phases observed in the second branch are similar to the ones observed by Khalak and Williamson for high $m^{*} \zeta$.

Fig. 9(c) shows the ratio between the current oscillating frequency and the body's natural oscillation frequency in still air ( $f^{*}$ defined in Table 1 ): even if the frequency ratio is close to 1 , as expected for tests in air with a higher value of $m^{*}$ than those performed in water, a small jump is seen in correspondence with the transition between the two branches. This is due to the added mass effects observable also in air because of the dimension of the model.

These results have been compared with those recorded at subcritical Reynolds Numbers using a smooth acrylic cylinder (see Table 2). An extensive experimental study was performed and documented in (Zasso et al., 2008; Belloli et al., 2012). Fig. 11 shows the cylinder response in the subcritical Reynolds Number range during progressive regime tests in terms of non-dimensional oscillation amplitude, lift coefficient, frequency ratio, and relative phase. The curves were obtained by increasing wind velocity for three different levels of non-dimensional damping (see Table 2). Fig. 10 compares the non-dimensional amplitudes, z/D, of the two studies in the map of the vortex (Williamson and Roshko, 1988). The figure also shows data from literature on high mass damping tests. The map of vortex was obtained at low Reynolds Numbers, but data from tests at high Reynolds Numbers (subcritical and postcritical) are included to make a comparison with the state of the art. As highlighted in Belloli et al., 2012, the tests performed in subcritical Reynolds Number conditions shows some significant similarities with the ones reported in literature relating to lower Reynolds Numbers.

It is possible to note that the $0.2 \mathrm{~m}$ diameter model in small structural damping condition $\left(\mathrm{Sc}_{1}\right)$ reaches very high oscillation amplitudes (up to $1.1 z / D$ ): in the range from $U / U_{\mathrm{St}}=1.15$ to $U /$ $U_{\mathrm{St}}=1.29$, a steady state condition similar to the well-known Upper Branch regime is achieved.

The branches observed at lower and higher $U / U_{\mathrm{St}}$ for the subcritical Re curve can be related respectively to the Initial and Lower branches mentioned in the literature (see (Belloli et al., 2012) for a complete description of the results). In particular the Initial Branch is very similar to the one described in the literature, while the Lower Branch shows more differences in terms of maximum amplitude reached and extension: it is narrower and the oscillation amplitudes are smaller. Steady state response curves obtained on smaller models at the Politecnico di Milano Boundary Layer Wind Tunnel (Belloli et al., 2003) show a wider Lower Branch at higher oscillation amplitude and hysteresis regions. In the authors' opinion, the dimension of the scaled model compared with the characteristic length of wind tunnel turbulence could be the reason for this difference. However, these flow characteristics do not seem to influence the Initial and Upper branches.

Comparing the experimental result obtained in subcritical and postcritical conditions in terms of non-dimensional amplitude, some differences can be highlighted: at high Reynolds Numbers the Upper branch cannot be experimented with; as pointed out above, this was intentional, with high damping introduced using two oil dampers. Moreover, at higher Re the lock-in region is anticipated at lower $U / U_{S t}$ and it is wider than in the subcritical Re condition, starting at about $U / U_{\mathrm{St}}=0.85$ and finishing at $U / U_{\mathrm{St}}$

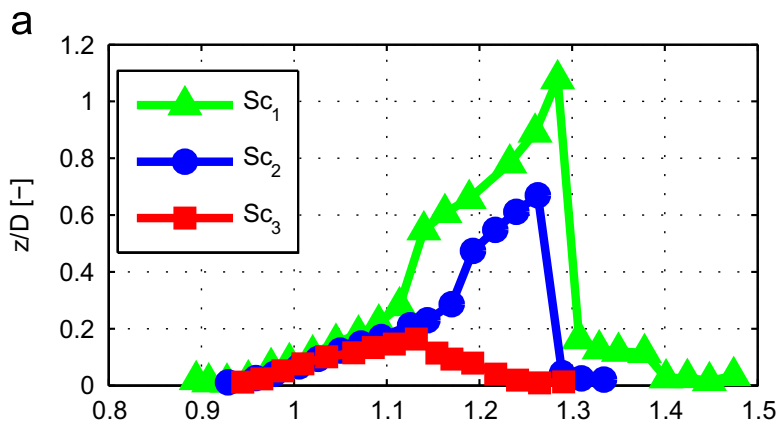

b
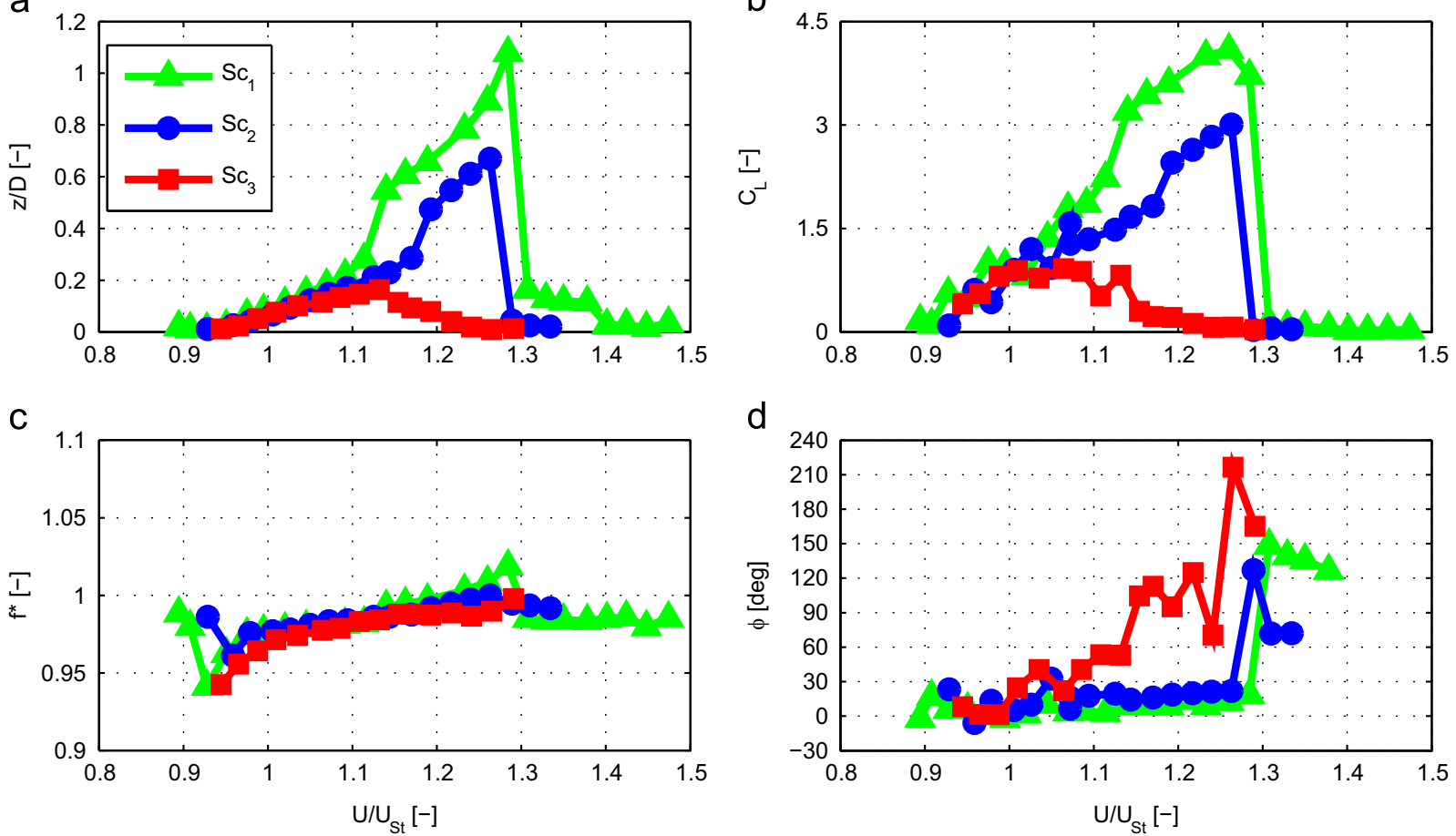

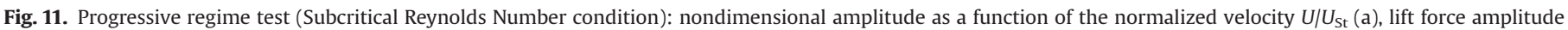
and phase (b and d), frequency ratio (c). The data are obtained by increasing the wind velocity for three different damping levels. 
$=1.4$. It is also to observe that Feng's results at $S c=2.20$ are very close to the present data at postcritical Re while they differ from subcritical Re at $S c=2.18$. The fact that we have three different curves having different maximum amplitude at the same Scruton Number, confirms that Scruton cannot be the only parameter that identify the maximum response of the cylinder in lock-in region. Also Williamson's equation (Eq. (1)) is not adequate to extrapolate the amplitudes in postcritical region. Experiments at high Reynolds Numbers and low Scruton are therefore needed to understand this dependence.

In spite of these differences between the results obtained at different Reynolds Numbers, some similarities were found in the flow-structure interaction in the Initial and Lower branches. Comparing the two models in terms of lift coefficient (the magnitude and phase of the synchronized lift component, Fig. 9 (b) and d) with Fig. 11(b and d)), the Initial Branch has a low oscillation amplitude, an increasing lift coefficient and a relative phase close to 0 deg for subcritical and postcritical Reynolds Numbers. The Lower Branch has low vibration amplitudes, small values of the lift coefficient and phase values around $90 \mathrm{deg}$. It is important to underline that the switch observed between the two flow conditions, where the lift coefficient magnitude (Initial Branch) and the lift coefficient phase (Lower Branch) respectively are effective, is similar both in the subcritical and postcritical conditions.

Moreover, in the Lower Branch it is possible to observe the appearance of unsynchronized vortex shedding: the lift force spectrum shows two specific frequencies, the cylinder's natural frequency $\left(f / f_{\mathrm{s}}=1\right.$ in Fig. 12$)$ and the Strouhal frequency $\left(f / f_{\mathrm{s}}=1.3 \mathrm{in}\right.$ Fig. 12).

Finally, it can be noted that, for the $0.2 \mathrm{~m}$ diameter model, no significant jump in the frequency ratio curves can be observed owing to the high value of mass ratio $m^{*}$ that makes added mass effects negligible (see Fig. 11 (c)).

\subsubsection{Surface pressure distributions}

The experimental set-ups made it possible to investigate the instantaneous pressure values on the cylinder. To compare the cylinder's behaviour as a function of the Reynolds Number, the Initial and Lower Branches were considered.
Fig. 13 shows the pressure distributions on the two models at the same non-dimensional amplitude in the Initial Branch but at a different velocity ratio. It is clear that the pressure distributions are similar, meaning that the interaction between the wind and the bluff body is substantially the same: the fluid exerts the same pressure on the cylinder surface.

The same situation is highlighted in Fig. 14 when the Lower Branch response is locked-in by the vibrating cylinder. As can be noted, the same vortex shedding modes are obtained for different $U / U_{\mathrm{St}}$ ratios. In this condition the pressure distributions have almost symmetrical distribution, with a small rotation of the stagnation point and hence low lift force values.

\subsubsection{Build-up tests}

Transitory tests were performed by releasing the cylinder from rest at a fixed wind velocity in the lock-in range and monitoring the vibration transient (increasing amplitude).

Fig. 15 shows the comparison between steady state responses obtained during progressive regime tests and the oscillation amplitudes obtained at the end of build-up tests in the postcritical Reynolds Number range. There is remarkably good agreement between the two sets of data. There are some differences in the region close to the maxima amplitudes where hysteresis was observed in the progressive regimes. Build-ups reached a higher amplitude level, suggesting that the hysteresis region could be larger than the one observed in the progressive regimes. The values obtained from transient build-up tests are representative of steady state conditions as showed in Zasso et al., 2006. Build-up tests are very useful for observing flow-structure interaction and understanding the mechanism of the power input by the wind. This analysis is important not only for understanding how the wind induces vibrations on the models, but also for seeing whether the interactions occur in a different way at different Reynolds Numbers. The significant quantities are the same as those observed for the steady state condition analysis: the dynamic response of the cylinder, the magnitude of the lift force and the relative phase between force and displacement. Some build-up tests performed both in the Initial and Lower branches have been considered to study how the transient develops in these two conditions and to highlight the differences.
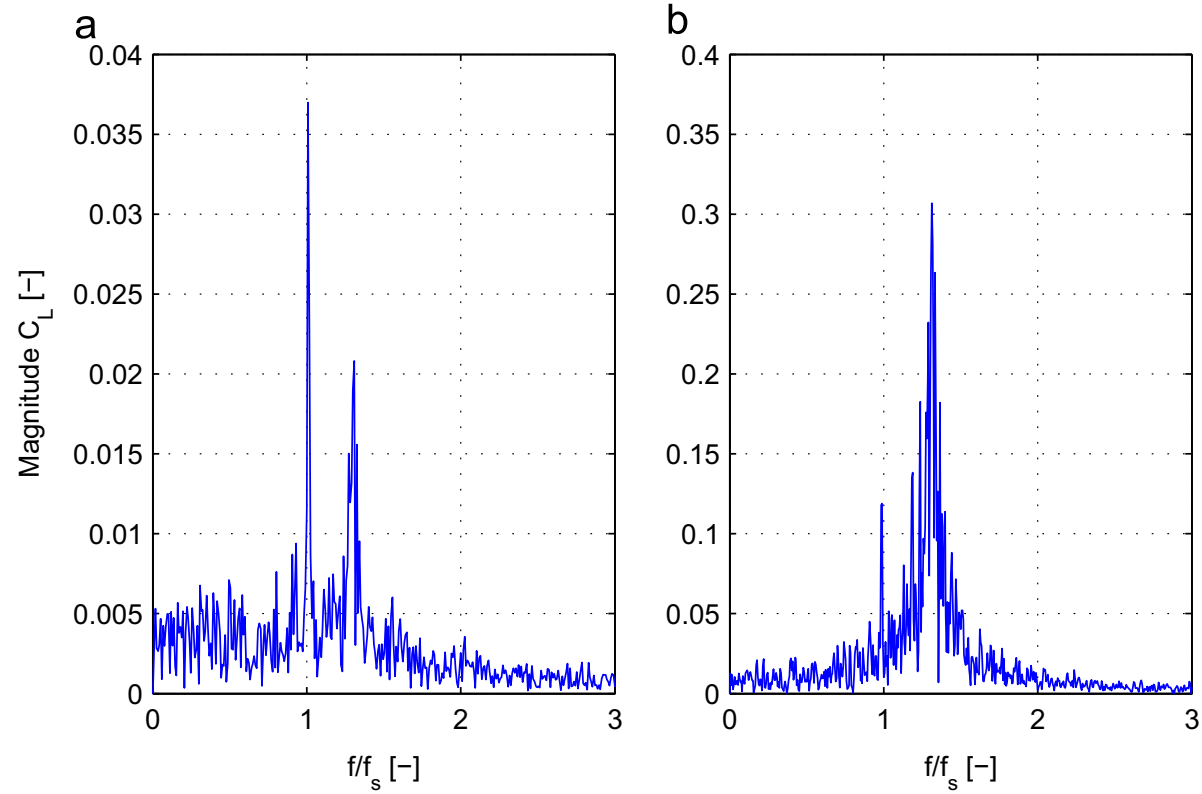

Fig. 12. Lift coefficient spectrum magnitude (a) Postcritical Reynolds Number $U / U_{S t}=1.3$ (b) Subcritical Reynolds Number $U / U_{S t}=1.31$. 


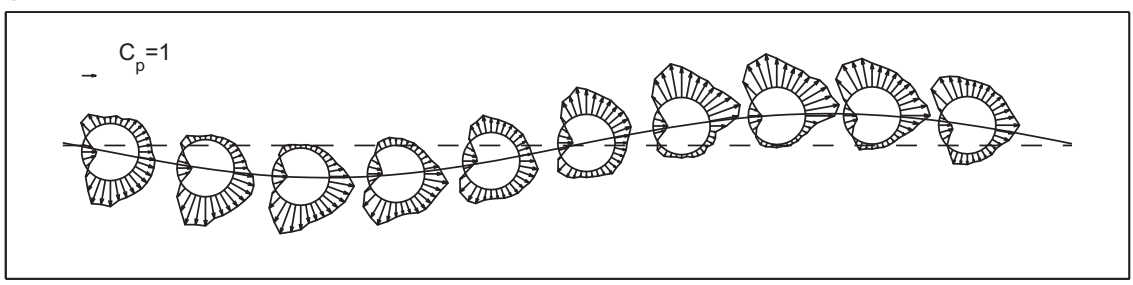

b

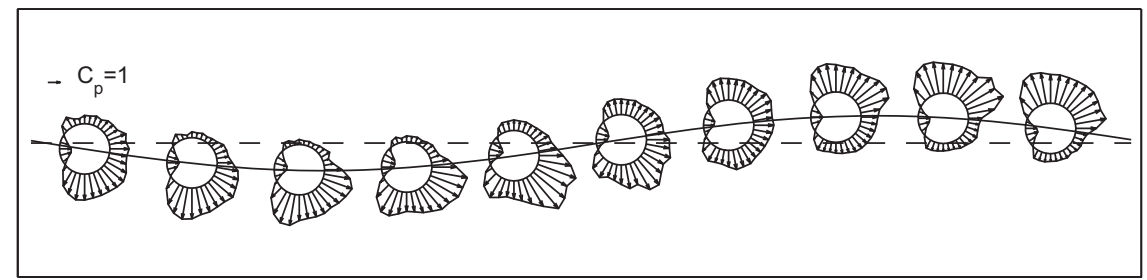

Fig. 13. (a) $z / D=0.16$ Postcritical Reynolds $U / U_{\mathrm{St}}=0.96$ (b) $\mathrm{z} / \mathrm{D}=0.16$ Subcritical Reynolds $U / U_{\mathrm{St}}=1.045$.

a

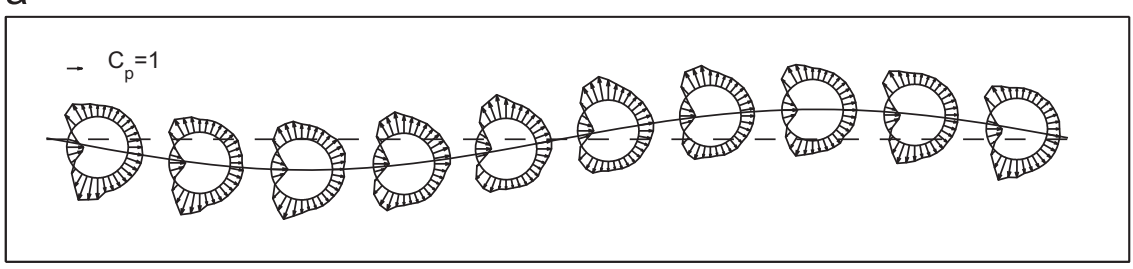

b

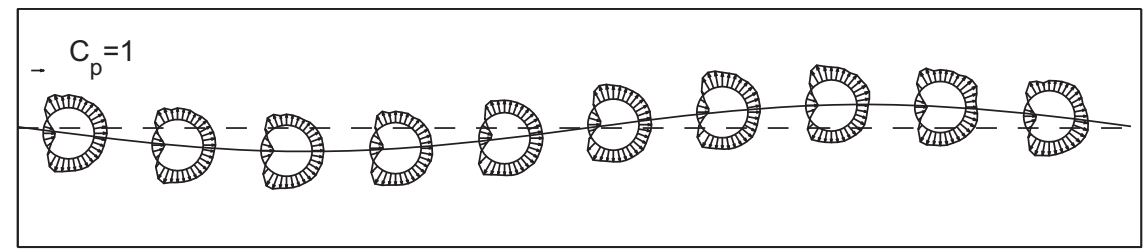

Fig. 14. (a) $\mathrm{z} / \mathrm{D}=0.145$ Postcritical Reynolds $U / U_{\mathrm{St}}=1.1$ (b) $\mathrm{z} / \mathrm{D}=0.145$ Subcritical Reynolds $U / U_{\mathrm{St}}=1.33$.

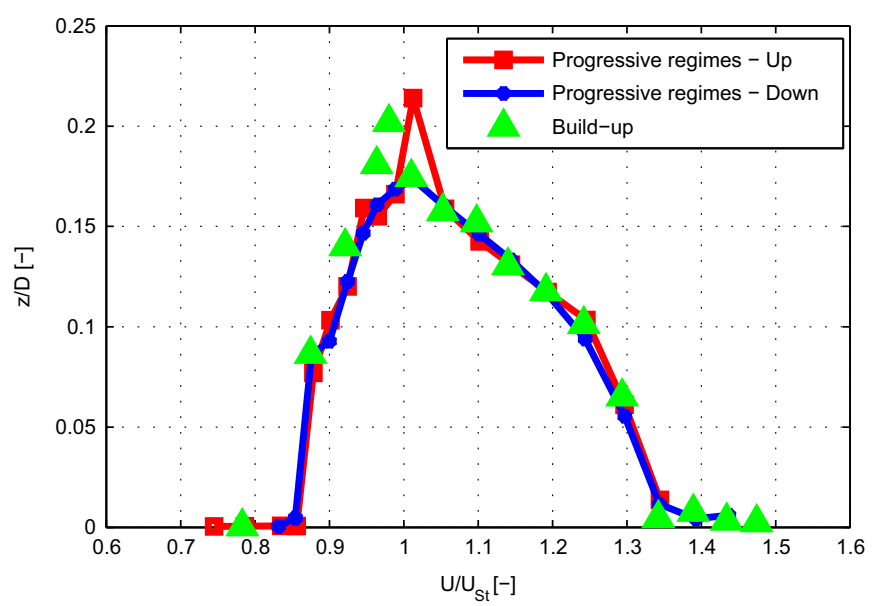

Fig. 15. Comparison between progressive regime tests and oscillation amplitudes reached at the end of the build-up (Rough cylinder, postcritical Reynolds Number range).

An example of a build-up test in the Initial Branch $\left(U / U_{\mathrm{St}}=0.98\right)$ at postcritical Reynolds Numbers is reported in Fig. 16 and 17. Fig. 16

shows the time histories of the cylinder's non-dimensional displacement and of the lift coefficient, while Fig. 17 shows the time-frequency analysis of the two signals. The harmonic components of the lift force and the cylinder oscillation amplitude at the cylinder's natural frequency were evaluated over about 10 cycles, using for the analysis a moving window with an overlap of $50 \%$. The trend of these components is reported as a function of time in Fig. 17(a). Fig. 17 (b) shows the relative phase between the harmonic compo-nents synchronous with the movement of force and displacement. The phase was calculated over 10 cycles.

Fig. 17 highlights how force and displacement evolve during the transient in the Initial Branch condition. Lift force and oscillation amplitudes grow almost simultaneously and the phase changes from about 90 deg to values close to $0 \mathrm{deg}$. Two behaviours can be identified: one at the beginning, where lift force and oscillation are small and the phase is close to $90 \mathrm{deg}$, and a second with high oscillation and force and low phase.

Considering these results in terms of energy input, two different mechanisms can be defined: at low amplitude the phase is very effective with small values of the force, while after transition, low values of phase coincide with large values of the force, meaning that the vortex shedding power input is proportional to the part of $\mathrm{C}_{\mathrm{L}}$ in phase with the cylinder velocity, that is, $C_{L} \sin (\phi)$, where $\phi$ is the phase angle. At low amplitudes the phenomenon is phase driven and at high amplitudes force driven.

This behaviour shows significant analogies with the behaviour observed in subcritical conditions for higher velocity ratios and for higher oscillation amplitudes corresponding to the end of the Initial Branch and to the Upper Branch, (Zasso et al., 2008). In particular, 

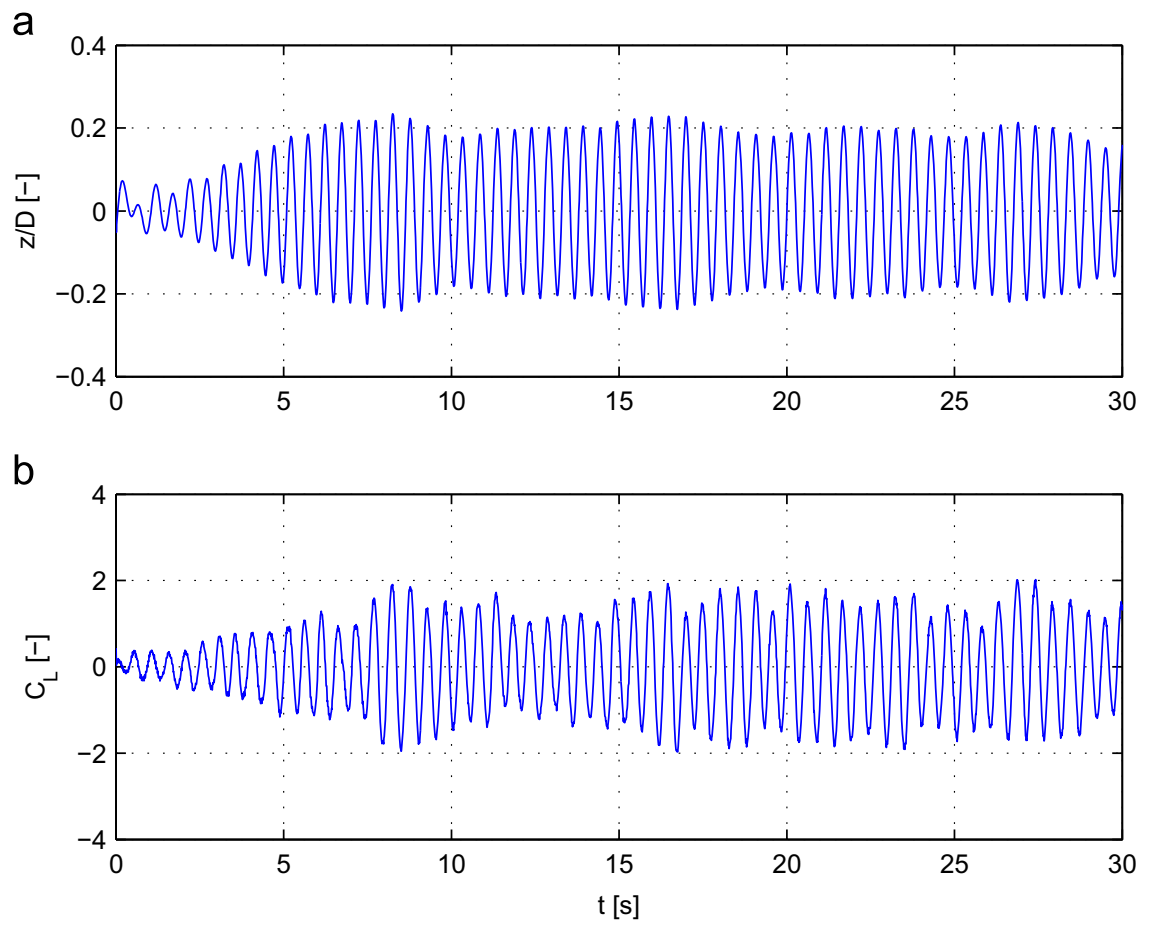

Fig. 16. Build-up test results (Postcritical Reynolds Number, $U / U_{\mathrm{St}}=0.98$ ): nondimensional displacement (a) and lift coefficient (b) time histories.
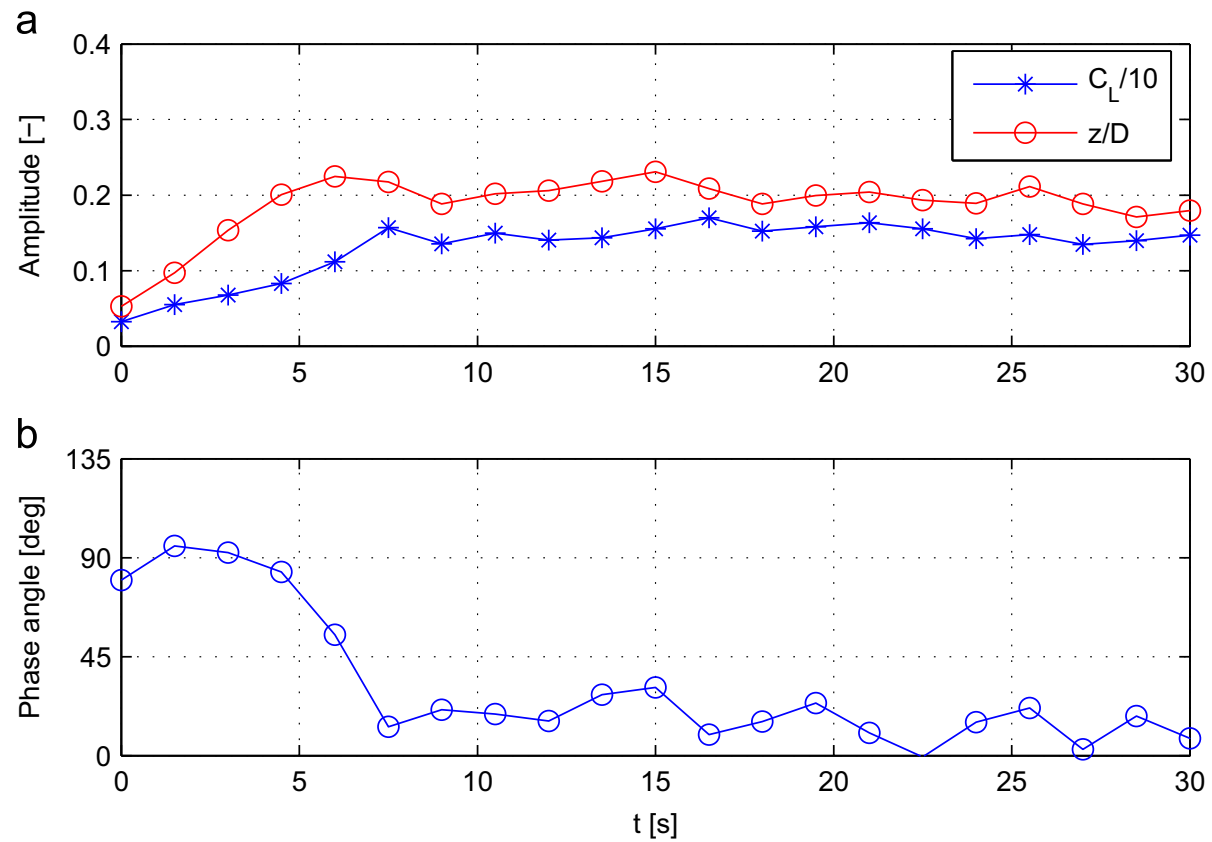

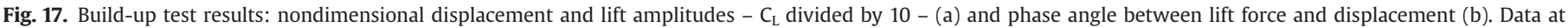
$U / U_{\text {St }}=0.98$.

the first state with low values of lift force and high phase and the regime condition after the transition with high lift force and small phase can be identified. A more detailed comparison between build-up tests in subcritical and postcritical conditions follows.

Fig. 18 and 19 show lift coefficient and cylinder nondimensional displacement during a build-up test performed at $U /$ $U_{\mathrm{St}}=1.05$ in Lower Branch conditions. Even when the cylinder oscillates at a large amplitude, the lift coefficient has very low values (see Fig. 18 (a) where the lift coefficient time history is presented using the same scale as Fig. 16(a)). Moreover, the relative phase is quite constant without sudden changes and is close to 90 deg. In this condition the power input mechanism is phase driven.
Build-up tests at four different velocity ratios are summarized in Fig. 20 and 21. They show the lift force magnitude and phase as a function of the non-dimensional oscillation amplitude. Fig. 20 refers to velocity ratios less than 1: a sudden transition in the phase occurs and the lift coefficient grows as the vibration amplitudes become larger. The threshold value of non-dimensional vibration amplitude when the rapid change occurs shifts to higher values, increasing $U / U_{\mathrm{st}}$.

The second group of data, $U / U_{\mathrm{St}}>1$, reported in Fig. 21, shows an almost constant value of both the lift force and the phase for all transient phenomena. The high value of the phase close to $90 \mathrm{deg}$ is typical of the Lower Branch in progressive regime tests. 

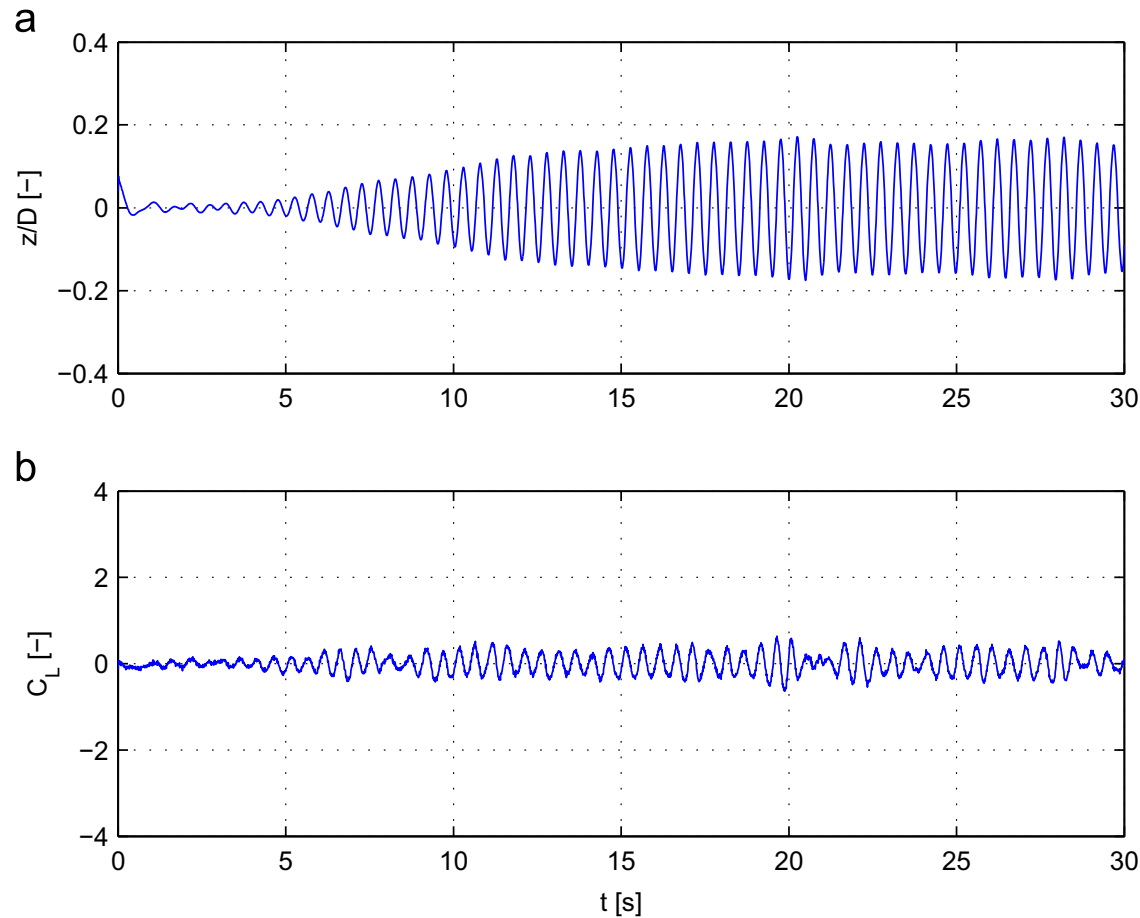

Fig. 18. Build-up test results (Postcritical Reynolds Number, $U / U_{\mathrm{St}}=1.15$ ): nondimensional displacement (a) and lift coefficient (b) time histories.
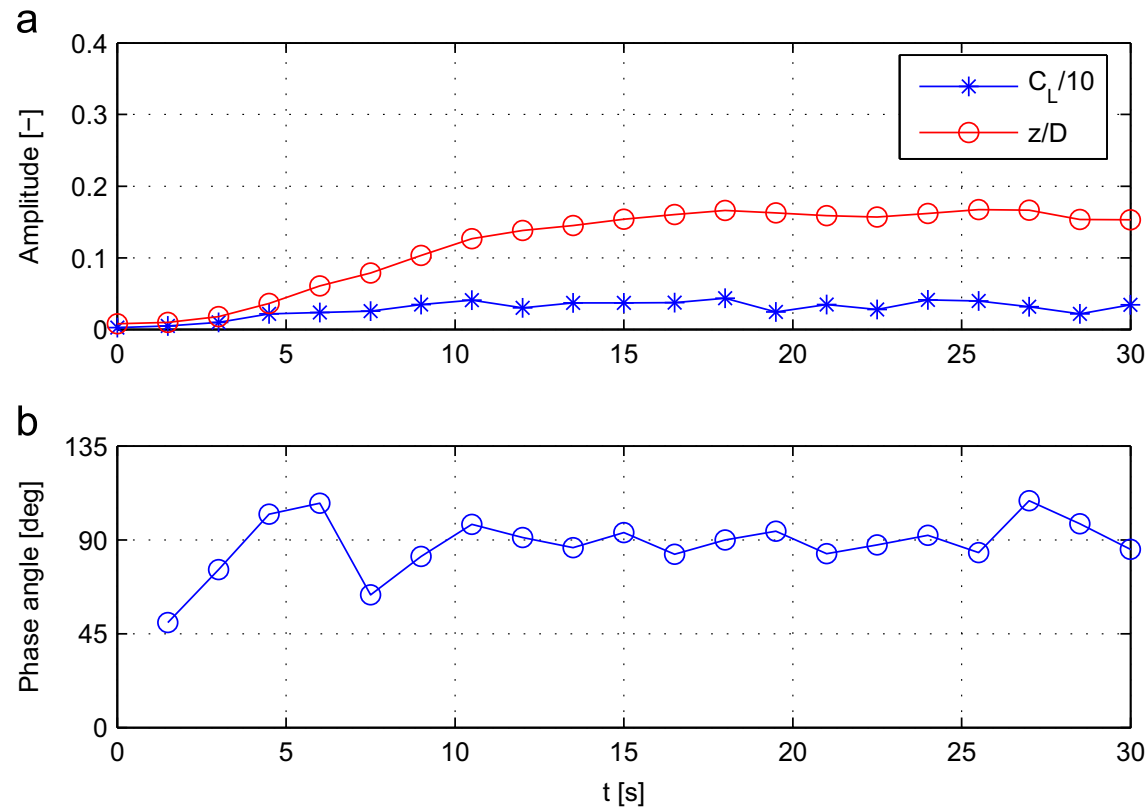

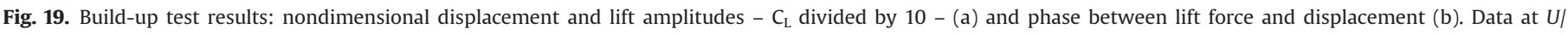
$U_{\mathrm{St}}=1.05$.

The results obtained in the postcritical Reynolds Number region were compared to those relative to the subcritical region reported in (Zasso et al., 2008). As pointed out above, there are many analogies between the two sets of data.

Fig. 22 shows lift force and relative phase as a function of cylinder non-dimensional displacement obtained using the smaller cylinder in Initial and Upper Branch conditions. As in the first set of data on postcritical Reynolds Number tests, at the beginning of the build-up, that is, for small cylinder displacements, low $C_{\mathrm{L}}$ values and phase close to $90 \mathrm{deg}$ are recorded until the amplitudes reach a threshold level function of $U / U_{\mathrm{St}}$. At the threshold level an abrupt change occurs and after the transition both phase and amplitude of
$C_{\mathrm{L}}$ show regular trends: the synchronized lift component grows with the vibration amplitude and the phase is positive and close to zero deg. Fig. 23 shows lift force and relative phase in the Lower Branch condition: as observed with the cylinder in this condition in the postcritical Reynolds Number range (see Fig. 21), the transient has low values of force and high values of phase (close to $90 \mathrm{deg}$ ). Build up tests confirm the results of the steady state tests highlighting that the mechanism of flow-structure interaction in the transient development is substantially similar in subcritical and in postcritical conditions. Power input calculation also confirms these observations: the set-up permits simultaneous measurement of the aerodynamic force and of the cylinder displacement, making 

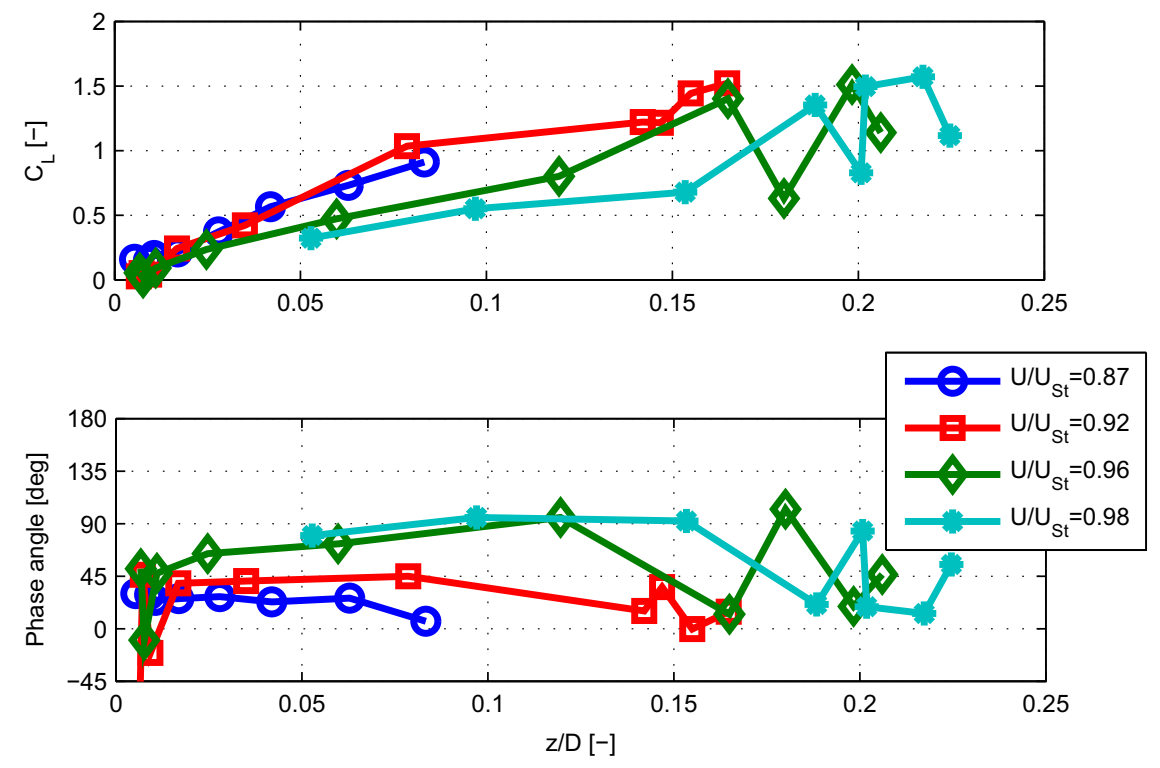

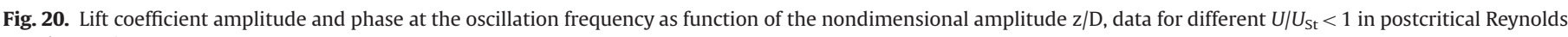
Number region.
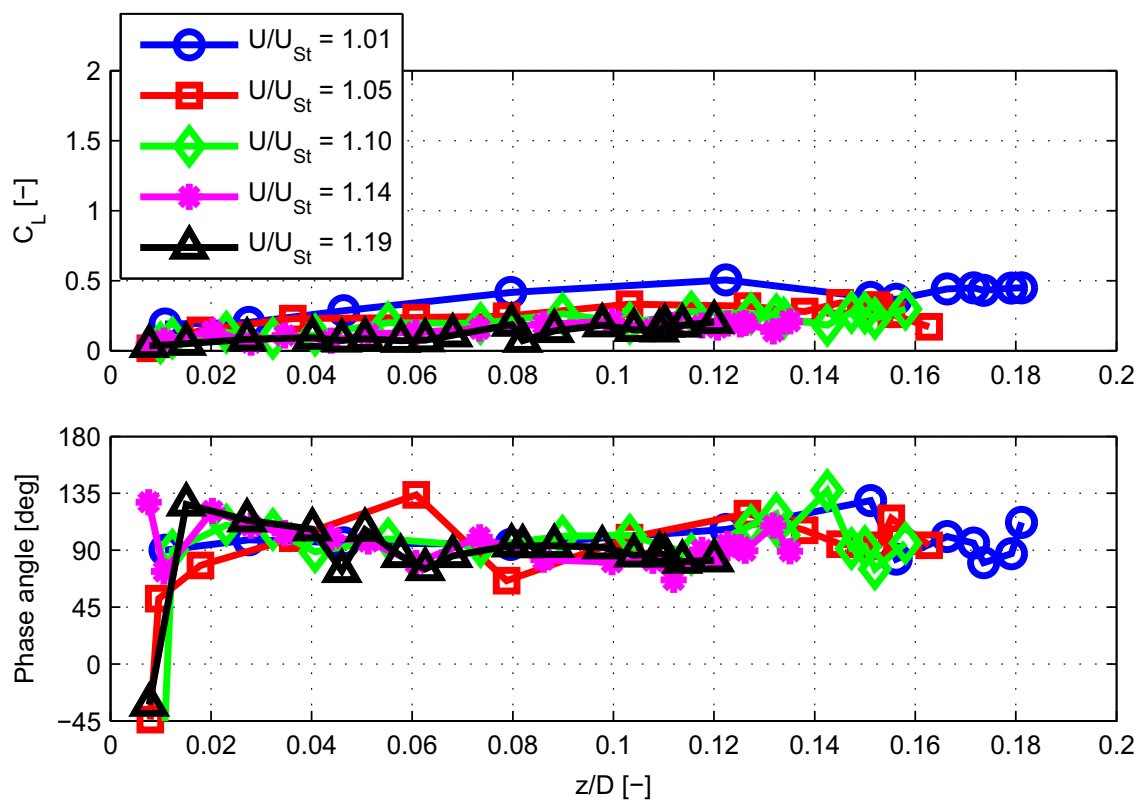

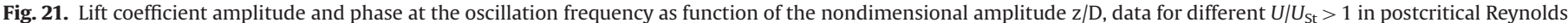
Number region.

it possible to define the power input by the flow into the mechanical system. Instantaneous power input was calculated as the product of lift force and oscillation velocity of the cylinder and was then integrated on a finite number of cycles. As reported in (Diana et al., 2008), good agreement between subcritical and postcritical regimes was found in terms of specific power input.

\section{Conclusions}

This article presents and discusses the main results of an experimental investigating on vortex induced vibrations of a circular cylinder in postcritical Reynolds Number conditions. Progressive regime and build-up tests were carried out, measuring pressure distributions and aerodynamic coefficients together with model displacement. The data collected were compared with the result of a similar research performed on a cylinder in subcritical Reynolds Number conditions, and the analogies observed between postcritical and subcritical phenomenology pointed out.

Progressive regime tests show similar Initial and Lower branches in both Reynolds conditions. It is expected that the postcritical cylinder would also show the Upper Branch regime, but its amplitude was limited by high structural damping to protect the equipment.

Build-up tests at lower $U / U_{\mathrm{St}}$ show a clear analogy between post and subcritical tests in the trend of lift magnitude and phase as a function of oscillation amplitude. In both cases, it is possible to identify the first state with its low values of lift force and high phase and the regime condition after the transition with high lift force and small phase. Increasing the $U / U_{S t}$ values, in the Lower Branch region, the two models show similar low values of lift force and high phase. 

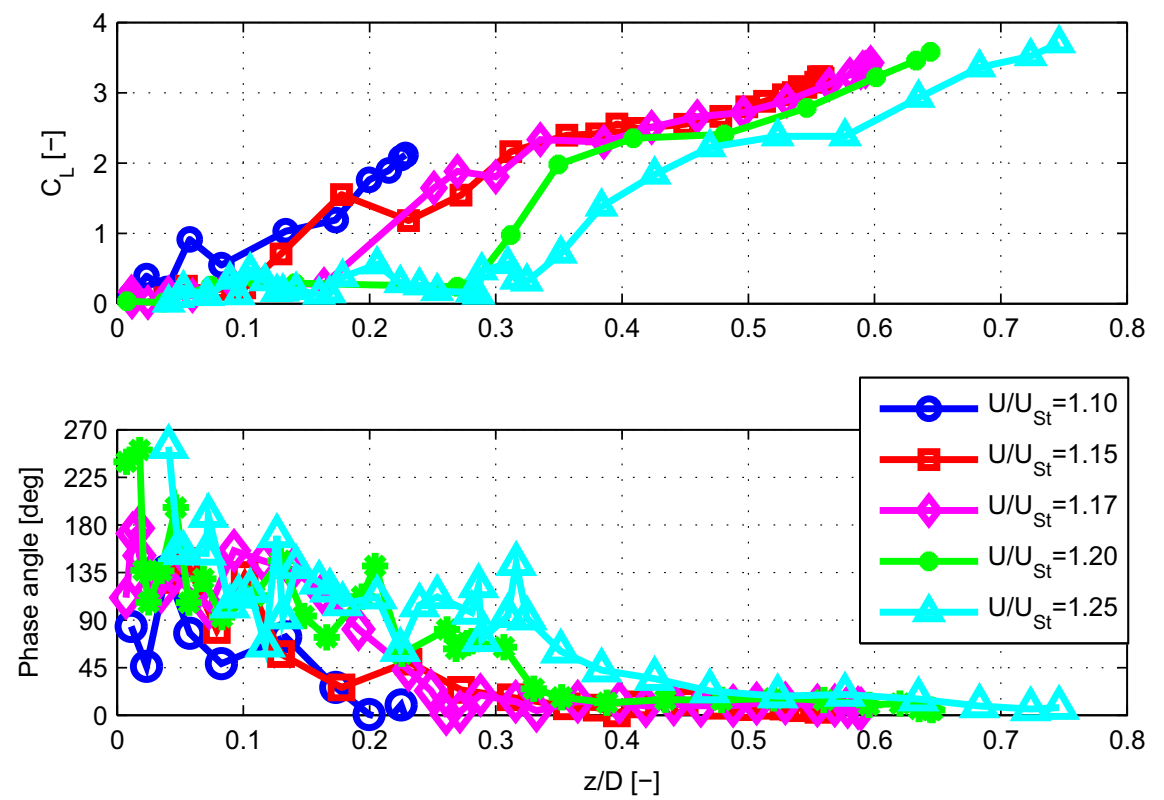

Fig. 22. Lift coefficient amplitude and phase at the oscillation frequency as function of the nondimensional amplitude $z / D$, data for different $U / U_{S t}$ relative to Initial and Upper branches in subcritical Reynolds Number region.
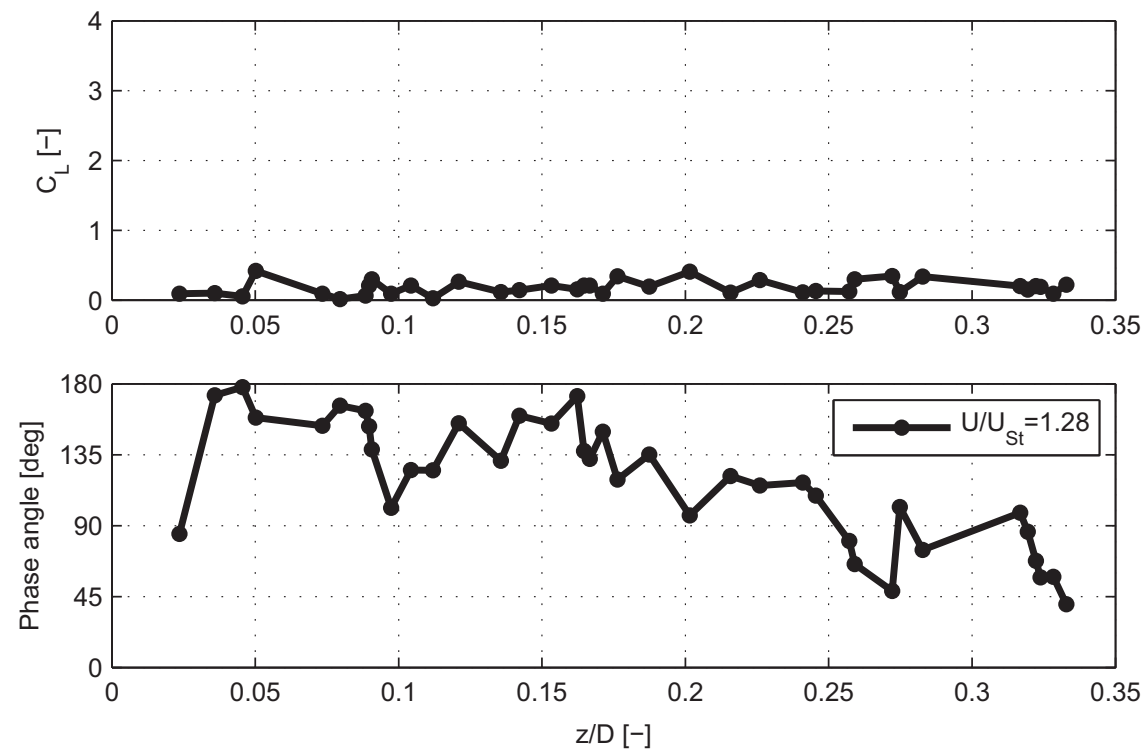

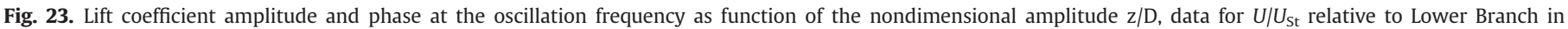
subcritical Reynolds Number region.

The pressure distribution analysis confirms the analogies previously highlighted for the two sets of data.

The behaviour observed leads to the conclusion that, after the disappearance of coherent vortex shedding and consequent vortex induced vibrations in the critical region, well-organized and coherent vortex shedding clearly reappears in the postcritical region with characteristics similar to those observed in the subcritical region.

Future steps in this research will be a study of the Upper Branch regime, up to now limited by test set-up. Amplitudes were controlled by adding damping to limit the forces on the suspension frame.

\section{Acknowledgements}

The Authors wish to acknowledge Dr. Guy Larose and Prof. Giorgio Diana for their valuable support in the research.

\section{References}

ASME, 2008. 27th International Conference on Offshore Mechanics and Arctic Engineering Volume 5: Materials Technology; CFD and VIV ISBN: 978-0-7918-4822-7 | eISBN: 0-7918-3821-8, http://dx.doi.org/10.1115/ OMAE2008-57045. Bearman, P.W., 1984. Vortex shedding from oscillating bluff bodies. Annu. Rev. FluidMech. 16, 195-222. Belloli, M., Cigada, A., Diana, G., Rocchi, D., 2003. Wind tunnel investigation on vortex induced vibration of a long flexible cylinder. Proceedings of the Fifth International Symposium on Cable Dynamics, Santa Margherita, Italy.

Belloli, M., Giappino, S., Muggiasca, S., Zasso, A., 2012. Force and wake analysis on a single circular cylinder subjected to vortex induced vibrations at high mass ratio and high Reynolds number. J. Wind Eng. Ind. Aerodyn. 103, 96-106.

Belloli, M., Giappino, S., Muggiasca, S., Zasso, A., 2007. Vortex shedding on circular cylinder at critical and postcritical Reynolds number. Proceedings of the Seventh International Symposium on Cable Dynamics, Vienna, Austria.

Brika, D., Laneville, A., 1996. A laboratory investigation of the aeolian power imparted to a conductor using a flexible circular cylinder. IEEE T. Power Deliver. $11,1145-1152$. 
Brika, D., Laneville, A., 1993. Vortex-induced vibrations of a long flexible circular cylinder. J. Fluid Mech. 250, 481-508.

Buresti, G., 1981. The effect of surface roughness on the flow regime around circular cylinders. J. Wind Eng. Ind. Aerodyn. 8, 105-114.

Carberry, J., Sheridan, J., Rockwell, D., 2001. Forces and wake modes of an oscillating cylinder. J. Fluids Struct 15, 523-532.

Diana, G., Belloli, M., Giappino, S., Muggiasca, S., 2008. Vortex induced vibrations at high Reynolds numbers. Proceedings of the Sixth International Colloquium on Bluff Bodies Aerodynamics \& Applications BBAA VI, Milano, Italy, pp. 1-10, ISBN 88-901916-4-3.

Diana, G., Falco, M., 1971. On the forces transmitted to a vibrating cylinder by a

blowing fluid - Experimental study and analysis of the phenomenon. Mecca-nica 6,

9-22.

Diana, G., Resta, F., Belloli, M., Rocchi, D., 2006. On the vortex shedding forcing

on suspension bridge deck. J. Wind Eng. Ind. Aerodyn. 94, 341-363.

Ding, J., Balasubramanian, S., Lokken, R., Yung, T., 2004. Lift and damping characteristics of bare and staked cylinders at riser scale Reynolds numbers, Proceedings of the Annual Offshore Technology Conference, vol 1. Offshore Technology Conference, Houston, TX; United States, pp. 518-526, ISBN 01603663.

ESDU, 1986. ESDU 80025. Mean forces, pressures and flow field velocities for circular cylindrical structures: single cylinder with two-dimensional flow, ESDU 80025, ISBN 085679308 6, ISSN 0143-2702, 0141-397X.

Feng, C.C., 1968. The measurement of vortex induced effects in flow past stationary and oscillating circular and D-section cylinders. Master's thesis. Univ. BC, Vancouver, Canada.

Govardhan, R., Williamson, C.H.K., 2000. Modes of vortex formation and frequency response of a freely vibrating cylinder. J. Fluid Mech. 420, 85-130. Govardhan,

R.N., Williamson, C.H.K., 2006. Defining the 'modified Griffin plot' in vortex-induced vibration: revealing the effect of Reynolds number using controlled damping. J. Fluid Mech. 561, 147-180.

Khalak, A., Williamson, C.H.K., 1999. Motions, forces and mode transitions in vortex-induced vibrations at low mass-damping. J. Fluids Struct 13, 813-851.
Pastò, S., 2008. Vortex-induced vibrations of a circular cylinder in laminar and turbulent flows. J. Fluids Struct 24, 977-993.

Raghavan, K., Bernitsas, M.M., 2011. Experimental investigation of Reynolds number effect on vortex induced vibration of rigid circular cylinder on elastic supports. Ocean Eng. 38, 719-731.

Roshko, A., 1961. Experiments on the flow past a circular cylinder at very high Reynolds number. J. Fluid Mech. 10, 345-356.

Sarpkaya, T., 2004. A critical review of the intrinsic nature of vortex-induced vibrations. J. Fluids Struct 19, 389-447.

Sarpkaya, T., 1979. Vortex-induced oscillations: a selective review. J. Appl. Mech. 46, 241-258.

Schewe, G., 1983. On the force fluctuations acting on a circular cylinder in crossflow from subcritical up to transcritical Reynolds numbers. J. Fluid Mech. 133, 265-285.

Williamson, C.H.K., Govardhan, R., 2004. Vortex-induced vibrations. Annu. Rev. Fluid Mech. 36, 413-455.

Williamson, C.H.K., Roshko, A., 1988. Vortex formation in the wake of an oscillating cylinder. J. Fluids Struct 2, 355-381.

Zasso, A., Belloli, M., Giappino, S., Muggiasca, S., 2008. Pressure field analysis on oscillating circular cylinder. J. Fluids Struct 24, 628-650.

Zasso, A., Belloli, M., Giappino, S., Muggiasca, S., 2006. On the pressure and force field on a circular cylinder oscillating in the lock- in region at sub-critical reynolds number. ASME Pressure Vessels and Piping Conference, Vancouver, BC, pp. 919-927.

Zasso, A., Larose, G.L., Giappino, S., Muggiasca, S., 2005. Effects of turbulence intensity and surface roughness on stays of cable-stayed bridges. Sixth

Inter-national Simposium on Cable Dynamics, Charleston, South Carolina.

Zdravkovich, M.M., 1997. (Foundamentals). Flow Around Circular Cylinders,

1. Oxford University Press, Oxford, New York 